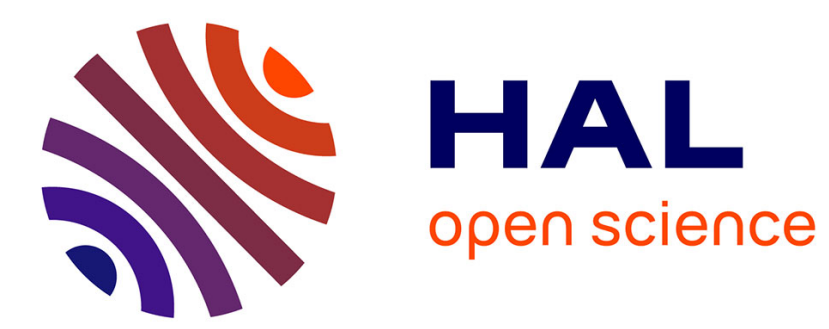

\title{
Structures à subordonnée comparative en français
}

Catherine Fuchs, Nathalie Fournier, Pierre Le Goffic

\section{To cite this version:}

Catherine Fuchs, Nathalie Fournier, Pierre Le Goffic. Structures à subordonnée comparative en français : problèmes de représentations syntaxiques et sémantiques. Lingvisticae Investigationes, 2008, LXXXI (1), pp.11-61. halshs-00340641

\section{HAL Id: halshs-00340641 https://shs.hal.science/halshs-00340641}

Submitted on 21 Nov 2008

HAL is a multi-disciplinary open access archive for the deposit and dissemination of scientific research documents, whether they are published or not. The documents may come from teaching and research institutions in France or abroad, or from public or private research centers.
L'archive ouverte pluridisciplinaire HAL, est destinée au dépôt et à la diffusion de documents scientifiques de niveau recherche, publiés ou non, émanant des établissements d'enseignement et de recherche français ou étrangers, des laboratoires publics ou privés. 
[paru dans Linguisticae Investigationes XXXI :1 (2008), pp. 11-61]

version préliminaire

\title{
Structures à subordonnée comparative en français : problèmes de représentations syntaxiques et sémantiques
}

\author{
Catherine Fuchs, Nathalie Fournier et Pierre Le Goffic \\ CNRS/ENS, Laboratoire LATTICE ; Université Lumière-Lyon2, Laboratoire ICAR ; \\ Université Paris3, Laboratoire LATTICE
}

\section{Introduction}

L'objectif du présent article est d'illustrer les principaux problèmes soulevés par l'analyse des structures à subordonnée comparative en français, et de présenter les différentes étapes nécessaires pour passer des représentations syntaxiques aux représentations sémantiques.

La représentation syntaxique concerne, d'une part, la structuration de la matrice et, d'autre part, celle de la subordonnée comparative. Nous proposerons une typologie des structures syntaxiques rencontrées, en examinant, d'une part, les structures corrélatives (en 'déclencheur + que') et, d'autre part, les structures en comme. La caractérisation de la matrice sera fondée sur la catégorie et la fonction du terme (jouant un rôle prédicatif) sur lequel s'ancre la subordonnée comparative. L'examen de cette dernière contrastera les structures verbales et averbales.

La représentation sémantique nécessite, comme on le verra, la reconstitution complète des prédications sous-jacentes à la proposition matrice et à la subordonnée. D'après les typologues (cf. L. Stassen 1985, B. Heine 1997, M. Haspelmath et O. Buchholz 1998), une phrase comparative comme Jean est plus grand que Marie comporte trois termes lexicaux et deux marqueurs grammaticaux. Jean est le terme 'comparé', Marie le terme 'standard' (ou encore 'comparant', càd. repère de la comparaison) et grand le terme 'paramètre' (ou 'prédicat') ; plus est le 'marqueur du paramètre' (ou 'marqueur de degré' - ce que nous avons appelé 'déclencheur') et que le 'marqueur du standard' (c'est notre 'terme en qu-').

Selon ce schéma, la comparaison opère entre deux termes (entités), au regard d'un paramètre (prédicatif). Dans un cas prototypique comme celui de l'exemple ci-dessus, l'identification du paramètre et des deux entités ne pose guère de problème. Mais tel n'est pas toujours le cas. Si l'on veut pouvoir couvrir la diversité des constructions comparatives, il est nécessaire de passer par deux étapes intermédiaires. La première consiste, nous le verrons, à épingler le constituant 'différentiel' présent dans la subordonnée, et la seconde à reconstruire le constituant 'parallèle' qui lui correspond dans la proposition matrice. Ce n'est que sur ces bases qu'il devient ensuite possible de caractériser les termes comparés et le type de comparaison.

Nous nous intéresserons ici, d'une part, à la comparaison quantitative et, d'autre part, à la comparaison qualitative en comme $(\mathrm{N}$. Fournier et $\mathrm{C}$. Fuchs sous presse a). Quand on opère une comparaison quantitative entre deux entités (plus/moins/aussi/autant/... + que), on mesure une intensité relative ou une quantité relative sur une échelle associée au paramètre. Quand on compare deux entités qualitativement (comme), on évalue une similarité fondée sur la manière (C. Fuchs et P. Le Goffic 2005). Nous ne traiterons pas ici de la comparaison qualitative fondée sur l'identité (même +que), l'altérité (autre +que) ou le type $(t e l+q u e)$ : sur ces constructions, voir M. van Peteghem (2000). 
L'article portera, dans l'ordre, sur les points suivants : (1) typologie des structures syntaxiques, (2) type de paramètre, (3) type de constituant différentiel, (4) type de constituant parallèle, (5) type de termes comparés, (6) type de comparaison et d'effets pragmatiques.

\section{Typologie des structures syntaxiques}

Nous considèrerons successivement la structure de la matrice A, puis celle de la subordonnée B. Nous ne développerons pas les différentes positions (nucléaires ou périphériques) de la subordonnée, ni ses diverses portées relativement à la matrice.

\subsection{Structure de la matrice de la comparaison}

Deux grands types sont à distinguer, selon que la matrice comporte un 'déclencheur' de la comparaison (plus, aussi, autre, ...) ou non. Dans tous les cas, la comparaison peut se jouer au niveau (dans le domaine syntaxique) d'une phrase (Marie est aussi jolie que ...; Marie est jolie comme ...) ou à un autre niveau (... une aussi jolie fille que...; ... une jolie fille comme ...).

\subsubsection{Avec déclencheur : ... $<>$ que}

Rappelons que le signe ' $<>$ ' note tout type de déclencheur : plus, moins, aussi, ainsi, de même, ...

On négligera ici les cas où comme se trouve associé à un déclencheur (type aussi grand comme ...). Ce type se rencontre dans la base en français 'moderne', mais dans des exemples qui ressortissent tous à un français archaïsant ou dialectal. Ces exemples ne soulèvent au demeurant aucun problème particulier de description syntaxique, et entrent sans difficulté dans les catégories proposées ici.

Le déclencheur peut porter de façon précise sur un terme se trouvant immédiatement à sa droite : un adjectif (plus grand), un adverbe (plus soigneusement), un nom (plus de $N$ ). Il peut porter, de façon, plus diffuse, sur un prédicat (V plein ou être sans attribut).

\section{a) $<>$ Adj que}

La portée du déclencheur sur un adjectif (ou plus exactement sur un Groupe Adjectival) représente le cas prototypique de la comparaison.

Le Groupe Adjectival peut être limité à un simple adjectif, comme il arrive le plus souvent, ou comporter des expansions: moins essentiel à la métaphysique, plus silencieusement amoureuse. Dans tous les cas, le déclencheur est considéré comme portant (syntaxiquement) sur le groupe adjectival dans son entier : c'est la subordonnée comparative, de son côté, qui peut induire une appréciation différenciée de l'impact sémantique de chaque sous-constituant.

Le Groupe Adjectival peut être constitué de l'amalgame d'une marque de comparaison (équivalente à un déclencheur) et d'un thème adjectival : meilleur, moindre, pire. Les adjectifs comparatifs autre, même, tel sont assimilables à des amalgames, en tant qu'ils participent à la fois d'un adjectif et d'un déclencheur de comparaison : du point de vue du présent article, autre, même et tel ont un fonctionnement analogue à celui de plus grand ou aussi bon, et ne nécessitent donc pas d'occuper une place particulière dans les exemples.

La prédication attributive portée par l'adjectif peut être assertée en tant que telle (dans une phrase attributive avec être ou équivalent), assertée secondairement (prédication seconde), ou enfermée dans un simple GN. En d'autres termes, l'adjectif (ou Groupe) support 
peut lui-même être attribut du sujet, ou être employé dans une autre fonction, dans une phrase ou dans un simple GN. Nous envisagerons successivement ces différents cas.

Les exemples sont présentés réduits à la matrice (sans la subordonnée), avec le pointeur $>$ devant le terme en $K w$ - introducteur de la subordonnée : cette présentation peut surprendre ou dérouter, mais elle a pour but de permettre une appréhension de la matrice indépendante de l'analyse de la subordonnée, cette dissociation des deux n'ayant pour but que de permettre en définitive une meilleure analyse de leur recomposition.

$\mathrm{a}_{1}$ ) Adjectif prédicatif : attribut du sujet

C'est le type Pierre est plus grand que Paul. En voici quelques exemples :

La vie était alors plus agréable > que (...)

Cette situation est moins angoissante $>$ que (...)

Le système phonétique des deux langues était aussi différent $>$ que (...)

Aristote n'est pas moins essentiel à toute métaphysique > que (...)

Les variantes de cette construction sont nombreuses. Le sujet peut être un GN ou n'importe quelle structure équivalente à un GN (Pronom, Groupe pronominal, Groupe Infinitival, Complétive, fragment en mention, ...) :

Être rayé de dessus l'État me seroit moins fâcheux > que (...)

La phrase peut être impersonnelle :

Il est aussi dangereux d'encourir sa faveur $>$ que (...)

La phrase peut comporter d'autres constituants (qui peuvent jouer un rôle dans le développement et l'interprétation de la comparaison (cf. infra §3.2.) :

\section{Il paraît encore plus grand mort $>$ que (...)}

(plus porte sur l'adjectif prédiqué grand, cette prédication s'accompagnant par ailleurs d'une prédication seconde, à valeur circonstancielle).

Dans le cas où le déclencheur est placé à droite (et non à gauche) de l'adjectif attribut, comme dans :

\section{Ce n'est pas obscur autant $>$ que (...), l'âme d'un chien}

nous considérons que le déclencheur porte en fait sur toute la relation prédicative (cf. cidessous), davantage que sur la qualité attribuée.

$a_{2}$ ) Adjectif prédicatif : attribut de l'objet

La prédication attributive peut se réaliser dans une structure d' « attribut de l'objet », autour de verbes du type trouver, imaginer, se montrer:

J'imagine cette ville beaucoup plus sombre > que (...)

[de la vieille Europe,] où l'homme s'est montré bien plus destructeur $>$ que (...) 
Relèvent également de cette catégorie les cas où l'adjectif est simplement attribut accessoire (le verbe n'étant pas par lui-même générateur d'une relation attributive) et non essentiel. On a alors une prédication seconde liée, comme dans :

Elle s'éveillait aussi lucide > que (...)

$\left.a_{3}\right)$ Adjectif prédicatif : adjectif prédicat second (détaché)

La prédication peut se réaliser sous forme de complément détaché, à l'initiale de l'énoncé, ou en position finale :

aussi simple et aussi novice > que (...), je ne restai pas même affriandé de jolies femmes.

Pauline était venue, plus maquillée et plus onduleuse $>$ que (...)

$a_{4}$ ) Adjectif épithète

La prédication attributive peut se réduire à une simple épithète de surface, antéposée ou postposée à un $\mathrm{N}$ dans un GN (une aussi jolie fille que Marie ; une fille aussi jolie que Marie), liée ou détachée (cette fille, aussi jolie que Marie ...).

Le GN peut lui-même remplir différentes fonctions (qui conditionnent en partie les possibilités ouvertes dans la subordonnée). Il peut être attribut du sujet :

.... parce que la pluralité étoit un péché plus grand $>$ que (...)

complément d'objet direct :

Les méchants princes souffraient, dans le Tartare, des supplices infiniment plus rigoureux $>$ que (...)

Les laides qui plaisent allument de plus furieuses passions et de plus violents engouements chez les hommes > que (...)

On prenait dans la rue une femme, une passante, une bourgeoise, aussi peu vieille et aussi peu laide > que (...)

sujet :

Une odeur fade [...], moins atroce > que (...), flotte en effet autour de cet homme chétif, dévoré d'une austère vie.

inséré dans un GPrép complément d'objet indirect :

Avez-vous jamais oü̈ parler d'une étoile si brillante $>$ que (...)

[notre situation ...,] qui tenait à des causes bien plus générales $>$ que (...)

inséré dans un GPrép essentiel derrière être :

L'âne est d'un naturel aussi sensible, aussi patient, aussi tranquille $>$ que (...)

L'affaire n'est pas en meilleurs termes > que (...)

inséré dans un GPrép circonstanciel :

Souvent un jeune homme quitte sa promise pour une jeune fille plus riche $>$ que (...) 
Pour nous en convaincre par un exemple encore plus frappant $>$ que (...)

inséré dans un GPrép complément de nom (le GN superordonné pouvant avoir lui-même une fonction quelconque) :

Donnons-lui le reste / D'un jour autant heureux > que (...)

Syllia et sa bonne amie s'assirent sans façon sur les rochers blancs, devant les flots d'un azur plus foncé mais aussi immobile et magnifique > que (...)

ou être un simple $\mathrm{N}$ apposé :

[Ahmès ...], Nubien plus noir > que (...) [était bon comme une nuit de sommeil]

b) $<>\operatorname{Adv} q u e$

Le déclencheur peut être placé à gauche d'un modifieur adverbial du prédicat, auquel cas il est légitime de considérer qu'il est lui-même un modifieur de ce modifieur :

Je lui ai répondu aussi tranquillement $>$ que (...)

La Reine sa mère ne l'aimoit pas plus tendrement $>$ que (...)

Un modèle est plus clairement exprimé par le pinceau abondant et facile d'un coloriste

$>$ que (...)

Le prince fut aussi vite calmé > que (...)

A signaler le cas des adverbes de manière avec être, sémantiquement et fonctionnellement équivalents à des adjectifs attributs (la 'manière d'être' vaut qualité) :

Où seriez-vous mieux >que (...)?

c) $<>$ de $\mathrm{N}$ que

Le déclencheur peut porter sur un Nom (plus d'élèves, moins de temps). Le GN peut remplir diverses fonctions dans la phrase. Il peut être complément d'objet direct :

Le vieil Ennius disait déjà qu'il avait autant d'âmes > que (...)

Votre article sur Volupté, mon cher Hippolyte, a rencontré autant d'admirateurs que (...)

J'ai vu mourir presque autant de lois scientifiques > que (...)

ou complément prépositionnel :

L'humanité se compose de plus de morts > que (...)

[ce] que je ne pouvais prendre avec autant de philosophie $>$ que (...)

d) $\mathrm{V}<>$ que

Le déclencheur peut ne pas avoir de portée stricte sur un terme situé immédiatement à sa droite. On considère alors qu'il modifie le prédicat. Il est alors délicat de déterminer a priori sa portée, entre une portée large sur le prédicat dans son ensemble (valant unité sémantique insécable), et une portée plus étroite, sélectionnant tel constituant particulier de cet ensemble. La place du déclencheur est ici de première importance, même si elle ne permet pas de régler univoquement le problème à tout coup. 
Dans certains exemples, le déclencheur joue un rôle de constituant essentiel (actanciel) : ainsi quand il est complément d'objet direct d'un verbe comme faire ou risquer :

$$
\begin{aligned}
& \text { N'ai-je pas fait plus }>\text { que (...) ? } \\
& \text { Nous avons produit autant }>\text { que (...) }
\end{aligned}
$$

Mais dans la majorité des cas le déclencheur est un modifieur du prédicat. Selon la constitution de celui-ci, et l'agencement linéaire de ses constituants, le déclencheur peut occuper des positions diverses (qui peuvent le séparer de la subordonnée qu'il déclenche). Le déclencheur peut se trouver entre auxiliaire et verbe auxilié :

$$
\text { [un garçon] qui avait autant voyagé > que (...) }
$$

derrière le verbe (sans compléments) :

Elle ne s'effraya pas autant $>$ que (...)

Depuis que tu es mon rom [homme] pour tout de bon, je t'aime moins $>$ que (...)

entre le verbe et ses compléments :

L'absence est aussi bien un remède à la haine > que (...)

Les Barbares aiment moins ce qui est beau > que (...)

ou après les compléments du verbe :

Le comment intéresse ces savants davantage > que (...)

Leur aisance à s'exprimer en français surprenait André Lhéry autant $>$ que (...)

Il peut aussi être détaché (avec sa subordonnée) :

Pas plus > que (...), Maléna n'avait trouvé d'objection à ce mariage avec un cousin âgé

L'île de Sein, ni plus ni moins >que (...), avait été protégée par Dieu

Une construction particulièrement fréquente est à signaler: le tour aimer mieux (souvent renforcé par un second déclencheur plutôt), et son quasi-équivalent préférer (avec ou sans plutôt) :

J'aime mieux qu'il soit exposé au danger $>$ que (...)

Il préfère y étaler son intelligence $>$ que (...)

e) $\hat{E}$ tre $<>$ que

Le déclencheur peut enfin apparaître sans support, dans l'environnement du verbe être, dans des emplois de caractère plus ou moins métalinguistique. A partir des cas où il s'analyse comme attribut :

[que] ces abstractions étaient moins > que (...)

Dix, c'est plus > que (...) 
il en vient à être senti comme formant avec que une sorte de locution. Ainsi plus que est proche (pour le sens) de très dans des emplois comme :

\section{Ma position peut devenir plus > que (...)}

Cet emploi se retrouve, sans verbe être exprimé, dans des exemples comme :

Il ne faut rien de moins $>$ que (...)

Cette nouvelle plus > que (...) [me surprit et m'étonna beaucoup.]

dans lesquels la relation attributive reste sous-jacente (rien qui soit moins que ...; nouvelle qui est plus que ...).

\subsubsection{Sans déclencheur : ... comme}

La comparaison en comme ne comporte pas de marqueur qui «préindiquerait» dans la matrice la comparaison, comme fait le déclencheur. La structure de la matrice sera donc envisagée en termes de structure syntaxique globale, sans préjuger d'une portée étroite ou large de comme. Mais les cadres d'analyse ne seront pour autant pas très différents: la comparaison, que ce soit en 'déclencheur + que' ou en comme, prend racine, dans une large mesure, sur les mêmes composants syntactico-sémantiques de la matrice.

On distinguera donc ici encore, d'une part, entre les prédications attributives et les autres, et, d'autre part, entre les prédications déployées dans le cadre d'une phrase verbale, et les prédications 'résorbées' elliptiquement dans un GN. La relation particulière qui unit comme et être exprimé ou sous-jacent, permettant l'expression de la manière d'être, sera mise en évidence.

a) Prédication attributive : Adj. comme

On rencontre fréquemment des comparaisons en comme à partir d'une prédication attributive dans la matrice. Les exemples ci-dessous sont présentés dans le même ordre que dans la section correspondante avec déclencheur (cf. supra, § 1.1.1. a).

$\left.a_{1}\right)$ Adjectif prédicatif : attribut du sujet :

Je suis bilieux >comme (...)

Il est faux > comme (...)

Avec comme ... en position détachée, la comparaison porte sur toute la relation prédicative :

Tu es saine et vaillante, > comme (...)

[S'il est vrai que] la religion soit nécessaire aux hommes, > comme (...)

$a_{2}$ ) Adjectif prédicatif : attribut de l'objet :

Il les évoquait enchaînés > comme (...)

$a_{3}$ ) Adjectif prédicatif : adjectif prédicat second (détaché) :

Amoureuse > comme (...), elle ne pouvait être capable de se dominer

Cela surtout lui manquait, dévoré > comme (...) du désir qu'on s'occupât de lui 


\section{$a_{4}$ ) Adjectif épithète}

Comme dans le cas des comparaisons avec déclencheur, la prédication attributive peut se réduire à une simple épithète de surface, dans un GN qui peut lui-même remplir différentes fonctions.

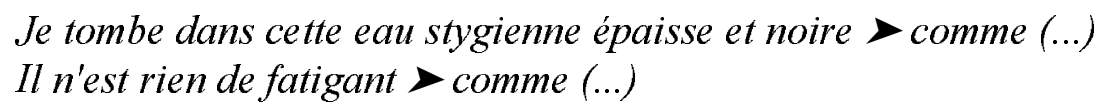

b) Prédication verbale + comme

La structure du prédicat peut être très variée, ainsi que l'ordre des constituants. La prédication peut être attributive, avec un attribut autre qu'un adjectif :

Je suis une bonne paroissienne > comme (...)

Le prédicat peut être un verbe intransitif,un verbe avec ses compléments, un verbe modifié par un adverbe :

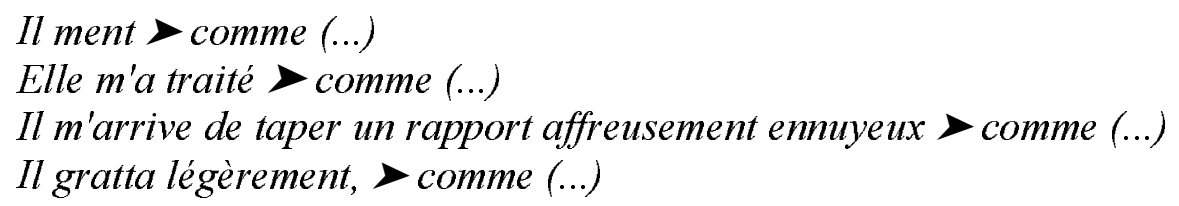

c) Prédication de manière d'être : Etre comme

On notera que la prédication de manière d'être revient à la qualité :

Chaque note est $>$ comme (...)

Votre père n'était pas alors, mon cher fils, >comme (...)

C'est une femme qui est très > comme (...)

d) Manière d'être implicite : N comme

Ce sont les nombreux exemples du type '(un) N comme X'. Le GN peut être sujet :

[Vous imaginez-vous, monsieur de Pourceaugnac, qu'] une fille > comme (...) [soit si affamée de mari ?]

complément d'objet direct :

[Il avait pourtant] un visage qui m'était familier, et $>$ comme (...)

attribut :

[C'est] un des désespérés de la vie > comme (...)

[C'était] un médecin original et $>$ comme (...)

ou derrière une préposition :

[Il ne voulait arriver qu'avec] des bottes >comme (...)

[C'est plein de] livres > comme (...) 


\subsection{La subordonnée comparative}

Deux grands cas de figure se présentent, selon que la subordonnée est une structure prédicative complète (ou d'apparence complète) autour d'un verbe, ou une structure non verbale.

\subsubsection{Subordonnée à structure verbale}

Si la subordonnée est une structure prédicative verbale, il y a lieu de distinguer selon le type de verbe : celui-ci peut être un verbe 'ordinaire' (être ou un autre verbe), ou parfois le verbe vicaire faire. Par ailleurs la subordonnée se limite très fréquemment à un verbe modal (attitude propositionnelle, verbe de parole, ...) et à son entourage immédiat.

a) Verbe plein

Le verbe peut être un verbe ordinaire, plein, avec le jeu complet d'actants qu'il comporte, et, le cas échéant, des circonstants :
(...) $>$ qu'elles ne chantaient
(...) > qu'il avait appris de parlers
(...) - que la Russie n'en connaîtra jamais
(...) $>$ comme elle respire
(...) $>$ comme on aime un vieil ami de quarante ans.

Le verbe peut être réduit à une forme auxiliée :

(...) > que bu.

Beaucoup des exemples ci-dessus présentent des anaphores d'actants ou de circonstants : la présence d'anaphores dans la subordonnée est un trait général caractéristique des comparaisons (intraprédicatives, du moins), pour des raisons sémantiques assez claires. C'est la même ligne qui, par delà des 'anaphores zéro', conduit en définitive à l'ellipse.

b) Verbe être. Exemples :

(...) - que la journée avait été chaude et animée

(...) > que ses résultats sont intéressants

(...) $>$ comme son devoir est de...

L'anaphore ne concerne pas seulement les actants et les circonstants, elle s'étend aux 'qualités'. Les exemples suivants exhibent clairement cette anaphore, réalisée soit par le pronom 'le', soit par 'zéro' :
(...) > qu'il l'est aujourd'hui
(...) $>$ que la vérité l'est de l'erreur
(...) > que ne sont les éléments d'Euclide à la géométrie
(...) - que Pompée n'a été en la sienne
(...) > comme elle était

(A côté du tour comparatif malheureux comme je suis (avec comme), le tour malheureux que je suis s'analyse comme relatif, et ne figure pas ici.) 
c) Verbe vicaire faire

Ce phénomène de l'anaphore est également sensible au niveau du verbe : la subordonnée reprend souvent le même verbe que la matrice, ou un verbe sémantiquement relié (synonyme, antonyme, ...).

Il existe également un verbe spécialisé dans les emplois anaphoriques : le verbe (dit 'verbe vicaire') faire, dont voici quelques exemples :

\section{(...) > que ne font d'ordinaire deux cousines \\ (...) $>$ que je ne fais ici \\ (...) > comme ferait un enfant avec son ongle \\ (...) comme il eut fait d'un papillon \\ (...) > comme j'eusse fait Shakespeare}

Pour le détail de ses emplois et valeurs (emploi avec ou sans le pronom anaphorique $l e$, emploi avec préposition $d e$, possibilités de reprise de être, etc.), voir N. Fournier et C. Fuchs (1999).

d) Verbe modal

Comme il a été indiqué ci-dessus, la subordonnée se limite très fréquemment à un verbe modal (attitude propositionnelle, verbe de parole, ...) et à son entourage immédiat : la subordonnée est souvent réduite à l'expression de cette modalité, le contenu propositionnel étant réduit à un le anaphorique de toute la proposition, ou à une anaphore zéro. En d'autres termes, la nature prédicative et verbale de la subordonnée ne signifie pas qu'elle n'est pas elliptique.

La modalité peut être clairement externe au contenu propositionnel (cf. les tours modaux impersonnels ou les sujets du type on), ou interne (du type vouloir). Il peut s'agir de verbes d'attitude propositionnelle :

\section{(...) > que ne l'imaginait l'enfant \\ (...) $>$ que vous ne pensez \\ (...) $>$ que je l'ai cru funeste}

de verbes de parole :

\section{(...)> que M. Roy ne le dit}

ou de verbes de modalité (proprement dite) :

$$
\text { (...) }>\text { que je ne devais }
$$

Plusieurs verbes d'attitude propositionnelle peuvent se cumuler, entre eux, ou avec le verbe vicaire :

$$
\begin{aligned}
& \text { (...) }>\text { que faire se peut } \\
& \text { (...) }>\text { comme on en voit qui prétendent faire }
\end{aligned}
$$

\subsubsection{Subordonnée non verbale}

Le plus souvent la subordonnée ne comporte pas de verbe. Elle se compose alors d'un terme (voire de plusieurs termes) ressortissant aux catégories syntaxiques et sémantiques suivantes : 
terme actanciel (GN ou équivalent), terme circonstanciel (Adverbe ou équivalent), ou terme de qualité (Adjectif ou équivalent).

a) Un terme actanciel : GN ou équivalent

C'est le cas type de la comparaison, abondamment représenté dans les exemples forgés des grammaires et des dictionnaires, comme dans les sources textuelles où ils s'alimentent :
(...) $>$ que le fond de mon cœur
(...) > que Cervantès
(...) $>$ que les autres coupables d'une condition privée
(...) > comme un jeton
(...) comme tout le monde
(...) comme la main

La question de la détermination du nom, importante pour l'interprétation, appelle une étude particulière. Le terme actanciel peut être un équivalent du nom - qu'il s'agisse de Pronoms et Groupes pronominaux variés :
(...) > que personne
(...) > comme tout

d'un Infinitif (ou Groupe Infinitival) :

(...) > qu'être effacé de votre mémoire

(...) comme de danser en comptant ses pas

de termes ou fragments divers utilisés en 'mention' :

(...) > que mon, ton, son, etc.

(...) comme Amitié, pitié, etc

de complétives (avec des difficultés spécifiques d'articulation sur que) :

(...) > que non pas qu'il a un peu trop voulu le copier

(...) > que qu'il ne meure

ou d'autres types de subordonnées :

(...) > comme qui dirait du vin

(...) > comme qui reviendrait de baptême ou d'enterrement

ou encore de l'expression d'une quantité :

$$
\text { (...) > que } 9
$$

(D'autres emplois de formations nominales, en particulier ça (dans pas plus que ça, grand comme ça) et probablement tout (gentil comme tout) relèvent sans doute du même mécanisme).

Un GN actanciel peut aussi être régi par une préposition. Voici des exemples de GPrép dont apparaît (en particulier par le trait '+ humain') le caractère actanciel : 
(...) $>$ qu'à l'attaque

(...) > comme à vous

b) Un terme circonstanciel : GAdv ou équivalent

Voici quelques exemples de subordonnée comparative réduite à un terme circonstanciel :

(...) > que jamais

(...) $>$ comme toujours

Les équivalents du Groupe Adverbial sont nombreux, que ce soit un Groupe Prépositionnel (à valeur circonstancielle) :

(...) > qu'en U.R.S.S.

(...) $>$ comme dans son enfance

un gérondif ou un infinitif circonstant :

(...) > qu'en vous attaquant

(...) $>$ comme pour se disculper

une subordonnée circonstancielle :

(...) > que si elle n'avait pas fermé l'oil

(...) > que quand c'est pour ruiner les choses mortelles ou tristes

(...) > comme si rien ne s'était passé depuis notre séparation

(...) > comme quand on est resté couché dessus trop longtemps

des groupes nominaux (temporels) à valeur de complément circonstanciel :

(...) > que l'année dernière

ou autres :

(...) comme inspiré par elle

(...) $>$ comme allant au-devant d'une question de ma mère

c) Un terme de qualité : GAdj ou équivalent

La subordonnée comparative peut consister en un terme marquant une qualité :

(...) que voluptueuse

(...) $>$ comme faible

L'adjectif de la subordonnée peut également être un adjectif modal, forme réduite de la prédication verbale modale évoquée ci-dessus (cf. supra, §1.2.1. c) :

(...) > que possible

d) Deux termes

La subordonnée comparative peut comporter deux termes. 
$\left.d_{1}\right) 2$ termes actanciels. Exemples :

(...) $>$ qu'un prêtre son bréviaire

(...) > comme un frère aîné une petite sæur sans amant.

(...) > comme une goutte d'eau à une autre goutte d'eau

$\mathrm{d}_{2}$ ) 1 terme actanciel, 1 terme circonstanciel. Exemples :

(...) $>$ qu'ici la nature

(...) > comme Léandre quand il allait rejoindre Héro

$\left.\mathrm{d}_{3}\right) 2$ termes circonstanciels, la plupart du temps dissymétriques. Exemples :

(...) > qu'ici avec vous

(...) > que naguère chez ses parents

$\left.\mathrm{d}_{4}\right) 1$ terme actanciel, 1 terme de qualité. Exemple :

(...) > que la sienne douloureuse.

Il serait parfaitement concevable qu'une subordonnée comparative averbale comporte plus de deux termes, mais nos sources ne nous en présentent pas.

\section{Le type de paramètre}

Nous abordons à présent des questions qui se situent à l'articulation de la syntaxe et de la sémantique. Pour la suite de l'exposé, nous nous limiterons aux cas où la subordonnée a une portée intraprédicative par rapport à la proposition matrice, càd. où elle s'articule sur le noyau prédicatif de celle-ci; nous laisserons de côté les cas de portée extraprédicative et exophrastique, qui ne seront signalés qu'en note (sur ces autres cas, voir N. Fournier et C. Fuchs sous presse b).

Dans le cas d'une corrélative en que, le paramètre est identifiable à partir du support du déclencheur présent en A : p. ex. grand dans Jean est plus grand que Marie. Dans les exemples prototypiques, le support du déclencheur coïncide avec le prédicat de A (c'est un verbe ou un adjectif attribut du sujet), ou avec un modifieur de ce prédicat (c'est un adverbe modifiant le verbe) ; le paramètre est alors immédiatement identifiable. Mais, comme nous le verrons, tel n'est pas toujours le cas.

Dans le cas d'une comparative en comme, l'absence de déclencheur rend la question plus délicate. Si l'on n'hésite guère dans un exemple comme Jean est beau comme un dieu, que faire d'exemples du type Je voudrais une robe comme la tienne ou Voici des roses telles que tu les aimes?

Du point de vue sémantique, le paramètre peut être défini comme ce que l'on quantifie ou que l'on qualifie. Nous défendrons l'idée qu'il s'agit toujours d'un élément ayant un fonctionnement de prédicat (gradable ou non, selon les cas). Nous considérerons successivement les cas où le paramètre renvoie à une qualité (adjectif ou adverbe), à une propriété (verbe en emploi non situationnel ou nom), ou à un procès (verbe en emploi 
situationnel) ${ }^{1}$. Puis nous aborderons les constructions où l'identification du paramètre est problématique.

\subsection{Une qualité}

Lorsque le support du déclencheur est un adjectif $^{2}$ (attribut du sujet, attribut de l'objet ou en prédication seconde) ou bien un adverbe modifieur du verbe, le paramètre (qui est le prédicat auquel correspond cet adjectif ou cet adverbe) renvoie à une qualité.

\subsubsection{Adjectif}

Dans des comparaisons quantitatives comme :

La vie alors était plus agréable qu'aujourd'hui

J'imagine cette ville beaucoup plus sombre qu'elle n'est femmes

Aussi simple et novice qu'auparavant, je ne restai pas même affriandé de jolies

le paramètre est le prédicat qui renvoie à la qualité 'être agréable', 'être sombre' ou 'être simple et novice'.

Le paramètre peut se trouver dédoublé en deux qualités :

Jeanne est plus jeune et plus jolie que Marie

ou désigner une qualité complexe exprimée par deux adjectifs coordonnés :

Claudel était beaucoup plus fin et intelligent que M. Roy ne le dit.

Employé avec un déclencheur quantitatif (plus, moins, ...), l'adjectif doit correspondre à une qualité gradable, càd. à laquelle est associée une échelle de degrés permettant une mesure relative. Sont donc exclus de cette configuration les adjectifs correspondant à une qualité non gradable, tels les adjectifs relationnels, les adjectifs de nationalité, etc. :

* Ta voiture est plus présidentielle que la mienne.

* Cet homme est moins anglais que sa femme

sauf emplois figurés, toujours possibles :

Je ne suis pas aussi féodal que mon cousin.

\footnotetext{
${ }^{1}$ Ces cas ne concernent que les constructions où la subordonnée a une portée intraprédicative. En cas de portée extraprédicative, le paramètre renvoie à l'assertion de la proposition : 'A est plus/moins/autant le cas que B (est le cas)', 'A est le cas, comme/de même que B (est le cas)'; en cas de portée exophrastique, il renvoie à l'énonciation de A : '(je dis que) A, comme/de même que X dit que B' : cf. N. Fournier et C. Fuchs (sous presse b).

${ }^{2}$ Comme on l'a vu au $\S 1$, on peut également trouver un groupe nominal ou un groupe infinitif en fonction d'attribut du sujet. On observe néanmoins que, dans ce cas, les subordonnées comparatives tendent à être comprises comme ayant une portée extraprédicative Ainsi C'est plus un amant qu'un mari ou L'important est plus de chercher que de trouver s'interprètent-ils préférentiellement comme signifiant 'Il est plus vrai/adéquat de dire (de lui) que c'est un amant que de dire que c'est un mari', 'Il est plus vrai/adéquat de dire que l'important est de chercher que de dire que l'important est de trouver'. Cette remarque vaut également pour les subordonnées en comme; ainsi : Je suis une bonne chrétienne comme tout le monde ('au même titre que tout le monde').
} 
En revanche, employés avec comme (marqueur qualitatif), tous les types d'adjectifs paraissent a priori possibles. On constate toutefois que lorsque comme suit un adjectif correspondant à une qualité non gradable, la lecture assignant une portée intraprédicative à la subordonnée semble bloquée :

\section{Cet homme est anglais comme sa femme}

s' interprète comme 'Cet homme est anglais, de même que sa femme (est anglaise)' et non pas comme 'Cet homme est anglais à la manière de sa femme/à la manière dont sa femme est anglaise'. La subordonnée est donc comprise comme ayant une portée extraprédicative, en sorte que le paramètre n'est pas la qualité exprimée par l'adjectif, mais toute la proposition matrice.

Considérons maintenant le cas où comme est employé avec un adjectif qui renvoie à une qualité gradable :

Jean est grand comme son frère.

Deux lectures sont possibles. Soit l'on retient A en entier comme paramètre (lecture extraprédicative de la subordonnée). La gradabilité de la qualité n'est alors pas prise en compte, et l'on ne compare pas la grandeur relative des deux frères: Jean est grand, comme/de même que son frère signifie seulement qu'ils sont grands tous les deux. Soit l'on sélectionne grand, qualité gradable, comme paramètre. Dans ce cas, comme (marqueur pourtant intrinsèquement qualitatif) se voit contribuer résultativement à l'expression d'une comparaison quantitative (d'égalité), du fait même du caractère gradable du paramètre; d'où la quasi-équivalence entre $\mathrm{Il}$ est grand comme son frère (littéralement 'il est grand à la manière de son frère') et Il est aussi grand que son frère. Il en va de même pour :

\section{Je suis bilieux comme tous les Diables}

qui, en lecture intraprédicative, équivaut à Je suis aussi bilieux que tous les Diables.

On pourrait sans doute justifier ce fonctionnement apparemment paradoxal en postulant, dans ces constructions 'adj. gradable + comme', l'existence d'un 'déclencheur zéro', qui ne serait pas sans rappeler les constructions anciennes du français (il est aussi/autant grand comme...) - voir l'article de B. Combettes dans le présent volume - ou les constructions actuelles d'autres langues romanes (espagnol tan ...como ; catalan tan ... com; occitan tan ... coma; portugais tão ... como).

\subsubsection{Adverbe}

Dans les comparaisons quantitatives, le support du déclencheur peut être un adverbe qui modifie le verbe. Le paramètre est alors la qualité à laquelle renvoie le prédicat qui correspond à cet adverbe - qualité qui elle-même qualifie le prédicat principal. Ainsi :

\section{Je lui ai répondu aussi tranquillement que la première fois}

qui se comprend comme une mesure quantitative d'égalité de degré de la qualité 'tranquillité (de la réponse)'. Ce qui suppose, là encore, que la qualité désignée par ce prédicat soit gradable. On notera au passage que si l'adverbe support du déclencheur modifie un adjectif, une ambiguité peut surgir ; ainsi, dans : 
Alberte, cette nuit-là, était plus silencieusement amoureuse que jamais

le paramètre est-il être silencieusement amoureuse ou bien être silencieux ?

En revanche, dans le cas de comme, un adverbe présent dans la proposition matrice ne peut jamais signaler le paramètre, car cet adverbe sature le prédicat, en sorte que la subordonnée introduite par l'adverbe comme ne s'interprète qu'avec une portée extraprédicative (l'entier de la proposition matrice constituant le paramètre). Ainsi :

\section{Je lui ai répondu tranquillement comme si de rien n'était}

ne peut signifier que 'Je lui ai répondu tranquillement, de même que je l'aurais fait si de rien n’était'. Il en va de même pour :

Tantôt je parcours des stades aux bords de la mer, tantôt je nage éperdument comme Léandre quand il allait rejoindre Héro

(remarquons qu'en l'absence de l'adverbe éperdument, la subordonnée pourrait au contraire se comprendre comme intraprédicative). Très souvent, l'interprétation extraprédicative est guidée par la présence d'une virgule marquant le détachement de la subordonnée :

\section{Il gratta légèrement, comme ferait un enfant avec son ongle}

- exemple que l'on peut opposer à Il gratta comme ferait un enfant avec son ongle, où l'absence d'adverbe dans la proposition matrice permettrait au contraire de retenir le verbe gratta comme indice du paramètre, autorisant ainsi une lecture intraprédicative ('il gratta à la manière d'un enfant avec son ongle').

\subsection{Une propriété}

Lorsque le support du déclencheur est un verbe en emploi non situationnel, le paramètre (qui est le prédicat auquel correspond ce verbe) renvoie à une propriété. Mais, comme nous allons le voir, un paramètre de type propriété peut également être construit à partir d'un support de déclencheur nominal.

\subsubsection{Verbe}

Dans une comparaison quantitative, des exemples comme :

\section{Il charme autant qu'il agace}

Il bégaie plus que sa søur

construisent une mesure relative sur une échelle de degrés (associée au prédicat 'charmer' ou 'bégayer', entendu comme une propriété) : on est ici dans l'intensité. Des exemples comme :

\section{Il lit plus qu'iln'écrit \\ Elle voyage moins que son mari}

construisent une mesure relative sur une échelle de degrés (associée au prédicat 'lire' ou 'voyager', entendu comme une propriété construite sur la base d'une activité itérative 'faire des lectures' ou 'faire des voyages') : on est alors dans la fréquence. Mais la quantification n'opère pas nécessairement en termes d'intensité ou de fréquence ; ainsi : 


\section{Jean mange plus que son frère}

se comprend-il comme 'il mange de plus grosses quantités'.

On remarquera que ces différents types de comparaison d'inégalité se paraphrasent de façon différente si l'on rapporte la propriété au sujet; on dira en effet C'est un plus grand charmeur et C'est un plus grand lecteur, mais C'est un plus gros mangeur. Sur cette opposition entre grand et gros dans les nominalisations agentives, voir H. Ulland (1993, § 4.3.1.).

Dans une comparaison qualitative, le verbe qui précède immédiatement comme livre le prédicat qui constitue le paramètre :

\section{Il ment comme il respire}

Chacun mangeait comme on mange en province

qualifient une manière de faire (une manière habituelle de mentir ou de manger) qui constitue une propriété.

Lorsque le verbe comporte des compléments, le paramètre est le prédicat correspondant au groupe 'verbe + compléments'. Ainsi :

Je t'aime moins que lorsque tu étais mon minchorro

Le comment intéresse ces savants davantage que le pourquoi

construisent une mesure relative sur une échelle de degrés associée au prédicat ' $t$ 'aimer' ou 'intéresser ces savants', entendu comme une propriété ('être amoureuse de toi', 'être intéressant pour ces savants'). De même :

\section{Il l'aime comme on aime à vingt ans}

qualifie la manière du prédicat 'aimer elle', en tant que propriété ('être amoureux d'elle').

\subsubsection{Nom}

Outre le verbe, un nom précédant comme peut également correspondre à un prédicat (de type être) constituant le paramètre. Ainsi :

\section{C'est plein de livres comme tu les aimes}

peut-il se gloser comme suit : 'c'est plein de livres qui sont comme tu aimes les livres', où comme est attribut de l'objet de la subordonnée ${ }^{3}$. Cette construction consiste à donc caractériser qualitativement un type particulier de $\mathrm{N}$, sur la base d'une propriété en être.

Ce mode de fonctionnement se rencontre surtout dans une perspective qualitative. Mais on trouve aussi quelques exemples de construction quantitative. Ainsi :

nuance plus nuance qu'on ne le pense

\footnotetext{
${ }^{3}$ Remarquons au passage que l'on retrouve un fonctionnement analogue avec tel que : Ce que veut Occide, c'est un communisme tel que [la Russie n'en connaitra jamais] se comprend comme '(...) c'est un communisme qui est tel que la Russie ne connaîtra jamais de communisme qui soit tel' (le terme tel jouant à la fois le rôle de déclencheur et de paramètre).
} 
qui revient à parler d'une 'nuance possédant effectivement la propriété 'être nuance' à un degré supérieur à celui auquel on pense ordinairement qu'elle la possède' ; dans cet exemple, la première occurrence du terme nuance est en emploi référentiel, alors que la seconde est en emploi prédicatif de paramètre.

\subsection{Un procès}

Lorsque le support du déclencheur est un verbe en emploi situationnel, le paramètre (qui est le prédicat auquel correspond ce verbe) renvoie à un procès. Ainsi :

\section{Elle ne s'effraya pas autant que...}

revient à nier une égalité de mesure sur une échelle quantitative d' 'effroi' en situation.

Il arrive que le support du déclencheur soit une séquence 'de + nom (ou groupe nominal)' objet du verbe. La perspective est alors toujours quantitative, qu'il s'agisse du prélèvement d'une quantité d'occurrences discrètes ou denses, ou bien du prélèvement d'une quantité compacte

Il a acheté plus de poires que...

Il a mangé moins de purée que... bonne.

(...) ce que je ne pouvais prendre avec autant de philosophie que faisait mon ancienne

Dans tous ces cas, nous considérons que le paramètre est le prédicat constitué par le groupe ' $\mathrm{V}+$ complément' (acheter des poires; manger de la purée; prendre avec philosophie). Ainsi dans :

\section{L'humanité se compose de plus de morts que de vivants}

le paramètre est le prédicat 'se composer de morts ${ }^{4}$ Dans les rares cas où le déclencheur porte sur une séquence de ce type en fonction sujet, comme dans :

Plus de femmes que d'hommes prennent le bus

un prédicat d'existence est sous-jacent : Il y a plus de femmes que d'hommes qui prennent le bus; cf. plus de peur que de mal.

Dans les comparaisons qualitatives en comme, la présence d'un verbe précédant comme permet d'identifier le paramètre comme étant le prédicat qui correspond à ce verbe :

\section{Elle m'a traité comme un chien}

qualifie un mode de 'traitement envers moi' particulier, càd. une manière de faire en situation. De même :

Elle m'accueillit comme si rien ne s'était passé depuis notre séparation

\footnotetext{
${ }^{4}$ Comme nous le verrons plus loin (cf. infra, § 5), dans le cas d'une construction de ce type, le paramètre est, plus exactement, le prédicat 'se composer de $\mathrm{X}$ ', assorti d'une variable $\mathrm{X}=$ 'morts'/'vivants' — l'indication de cette variable, qui n'est pas repérable au niveau de la seule matrice, étant donnée par le différentiel présent dans la subordonnée.
} 
qualifie la manière de 'l'accueil envers moi' dans la situation considérée.

\subsection{L'identification problématique du paramètre}

Contrairement à ces cas relativement simples, où le paramètre peut être calculé au sein même de la proposition matrice, on rencontre un certain nombre de structures dans lesquelles l'identification du paramètre pose problème et nécessite la prise en compte de la subordonnée. En effet, lorsque la proposition matrice a une structure plus complexe mettant en jeu plusieurs prédicats possibles, le paramètre ne coïncide pas nécessairement avec le seul support du déclencheur; dans ces cas, seule la reconstitution de la prédication sous-jacente à la subordonnée permet de décider de l'empan exact du paramètre.

Considérons par exemple le cas où le support du déclencheur est un adjectif en fonction d'épithète, càd. participant d'un groupe nominal complément du verbe de la proposition matrice :

Les méchants princes souffraient, dans le Tartare, des supplices infiniment plus rigoureux que...

Rien ne permet de dire si le paramètre est le seul prédicat 'être rigoureux (correspondant à l'adjectif support du déclencheur) au sein du GN objet, ou le prédicat 'souffrir des supplices douloureux' (correspondant au verbe et à son groupe objet) qui inclut, à un niveau syntaxiquement inférieur, le prédicat 'être rigoureux'. La première solution se vérifierait si la subordonnée était du type ... que cette simple tracasserie; en l'occurrence, c'est la seconde solution qui, dans le contexte de l'exemple originel, est la bonne, puisque la subordonnée dit ... que les autres coupables d'une condition privée.

Il faut donc pouvoir mettre en parallèle les divers prédicats de la proposition matrice avec la prédication (souvent à reconstruire) de la subordonnée. Cette reconstruction passe par l'identification du constituant différentiel dans la subordonnée et l'identification du constituant qui lui est parallèle dans la proposition matrice.

\section{Le type de différentiel}

Il s'agit d'identifier ce qui, dans la subordonnée B, est différentiel par rapport aux constituants de la proposition matrice A. Il faut donc repartir de l'analyse de B donnée au $\S 1$. supra: subordonnée verbale (verbe plein/verbe vicaire et anaphores/verbe modal) ou averbale (un terme - actanciel, circonstanciel, de propriété, réduction de $\mathrm{P}$ modale, mesure - ou deux termes).

Le différentiel ne coïncide pas nécessairement avec la totalité des éléments présents en $B$. En particulier, les anaphores et les vicaires ne sont pas des différentiels.

Du point de vue sémantique, le différentiel peut être une entité, une circonstance, un procès, une qualité, une propriété ou une modalité (de phrase ou de prédicat) - nous reprenons ici les catégories sémantiques dégagées pour le paramètre. Par ailleurs, le différentiel peut être simple ou complexe, càd. composé de plusieurs constituants, ce qui lui permet de dénoter, le cas échéant, une relation prédicative complète. Comme il a été dit plus haut, nous n'examinerons pas ici les cas où le différentiel est une situation (comparatives extraprédicatives) ou une énonciation (comparatives exophrastiques).

\subsection{Une entité}


Le différentiel peut être une entité humaine, non humaine ou abstraite, envisagée comme spécifique, non spécifique ou générique. Cette entité joue un rôle sémantique d'actant, qui ne peut être spécifié que relativement au prédicat sous-jacent de la subordonnée. C'est ainsi qu'une entité humaine spécifique comme son frère peut, selon les contextes, être support de propriété, siège du procès, agent, patient, bénéficiaire, etc. :
Jean est plus grand que son frère
Jean me plaît plus que son frère
Jean travaille plus que son frère
Jean aime plus sa sæur que son frère
Jean parle plus à sa sæur qu'à son frère.

On voit donc que, du moins quand le différentiel est un GN, on ne peut préjuger, dans le cadre de la seule subordonnée réduite, du rôle actanciel qui est le sien relativement à la structure comparative dans son ensemble.

Sur le plan syntaxique, le différentiel peut être le seul constituant (GN ou équivalent) de la subordonnée non verbale. Il peut aussi être l'argument d'un vicaire ou d'un verbe plein identique à celui de la matrice :

La Reine sa mère [...] ne l'aimait pas plus tendrement que faisait Anne d'Espagne

Il aimait Célestin comme on aime un vieil ami de quarante ans.

Quand l'entité est le patient d'un procès vicarisé par faire, il peut être construit directement ou au moyen d'une préposition :

Je saluai mon vieux maître comme j'eusse fait Shakespeare

Papa le saisit au vol, avec légéreté, comme il ê̂t fait d'un papillon

Cette dernière construction est devenue la construction standard en français moderne : voir $\mathrm{N}$. Fournier et C. Fuchs (1999).

Les comparatives à parangon ont prototypiquement pour différentiel une entité générique : faux comme un jeton, nu comme la main, douce comme une peau de pêche; parfois aussi une entité spécifique (désignée par le nom propre) à valeur exemplaire : fort comme Hercule, pauvre comme Job, plus espagnol que Cervantès.

\subsection{Une circonstance}

Le différentiel dénote une localisation spatiale ou temporelle. Ce peut être un complément circonstanciel :

\section{Le phénomène concentrationnaire en Chine est beaucoup moindre qu'en U.R.S.S}

Chacun mangeait comme on mange en province.

ou une subordonnée circonstancielle temporelle ou hypothétique (localisation fictive) :

Je t'aime moins que lorsque tu étais mon minchorro

Elle m'accueillit comme si rien ne s'était passé depuis notre séparation

ou bien un gérondif ou un infinitif circonstant : 
Les hommes risquent davantage en vous laissant vivre qu'en vous attaquant Il y a autant de faiblesse à fuir la mode qu'à l'affecter

ou encore un adverbe :

La vie était alors plus agréable qu'aujourd'hui

Je travaille mieux chez moi qu'ici

ou, enfin, une qualité :

Il est plus grand mort que vivant.

Parfois la localisation temporelle différentielle est marquée par la seule détermination du vicaire :

Marie est plus jolie qu'elle ne l'était.

Mais le plus souvent cette détermination temporelle est congruente avec la localisation temporelle marquée par le différentiel-circonstance :

A la fin du dix-huitième siècle, l'existence du ghetto est menacée comme elle ne l'a jamais été Dans cent ans l'homme sera-t-il moins malheureux qu'il l'est aujourd'hui ?

J'en parlerai (...) moins sobrement que je ne fais ici.

\subsection{Un procès}

Il s'agit des cas où la subordonnée comporte un verbe plein en emploi situationnel (dont le sujet est coréférent au sujet de la proposition matrice) :

Les petites Irlandaises dansaient mieux qu'elles ne chantaient Jean s'en alla comme il était venu.

3.4. Une qualité

Le différentiel est un adjectif, qui qualifie a priori une entité :

Cette situation est moins angoissante qu'absurde

ou un adverbe (ou équivalent) qui qualifie a priori un procès :

Il chante plus vaillamment que musicalement

Il répondit avec plus de véhémence que de réelle conviction.

\subsection{Une propriété}

Le différentiel est alors un verbe en emploi non situationnel (typiquement au présent générique) ou une notion :

Il ment comme il respire

(...) une femme plus que femme. 
3.6. Une modalité (de prédicat ou de phrase)

Si le différentiel est une modalité de prédicat, le verbe modal (pouvoir, devoir, vouloir ...) a un sujet coréférent au sujet de la proposition matrice et le prédicat modalisé est anaphorisé (le ou anaphore zéro) ou vicarisé :

Elle ne s'effraya pas autant qu'elle l'aurait dî

N'ai-je pas fait plus que je ne devais?

Si le différentiel est une modalité de phrase, le verbe modal (croire, penser, dire ...) a son propre sujet (distinct du sujet de la matrice) et la phrase modalisée est anaphorisée par le (ou zéro) :

La mer était plus impressionnante que ne l'imaginait l'enfant Je suis plus ferme que vous ne pensez.

Ce peut être aussi une modalité impersonnelle :

(...) une femme (...) aussi peu vieille et aussi peu laide que possible Ils sont (...) les gens comme il faut de la république.

\subsection{Le cas des différentiels complexes}

Le différentiel peut être composé de plusieurs constituants (on l'a vu au $§ 1.2 .2$. d supra), qui donnent lieu à diverses combinaisons sémantiques :

- deux entités, avec un contraste actanciel :

Pour que je la console comme un frère aîné une petite sœur sans amant

(que) l'expression de M. Arnauld soit autant différente de celle des Pères que la vérité l'est de l'erreur

Une démocratie imposée par la force (...) ressemble à la dictature comme une goutte d'eau à une autre goutte d'eau

(...) les hirondelles (...) plus fidèles à leur nid que l'homme à sa maison

- une entité et une qualité :

Ma vie était maintenant aussi sotte et ennuyée que la sienne douloureuse

- une entité et une circonstance :

(la vieille Europe,) où l'homme s'est montré bien plus destructeur qu'ici la nature

- deux circonstances :

Est-ce que je serai jamais si heureux qu'ici avec vous?

Quand tous les constituants de B sont des différentiels, cela construit une relation prédicative complète : 


\section{Schumann.}

Il m'arrive de taper un rapport affreusement ennuyeux comme un autre jouerait $d u$

On notera qu'un différentiel complexe entraîne souvent (surtout dans le cas d'un contraste entité/qualité) la présence d'un vicaire (être, faire), qui articule les deux constituants du différentiel :

La démonstration de M. Sudre est aussi ingénieuse que ses résultats sont intéressants.

Dans ce cas, le différentiel complexe peut s'enrichir d'un marquage contrastif sur le vicaire, d'ordre temporel ou modal :

La nuit fut aussi douce, aussi calme, que la journée avait été chaude et animée Elles se ressemblaient beaucoup plus que ne font d'ordinaire deux cousines Donnons-lui le reste/D'un jour autant heureux que je l'ai cru funeste.

\subsection{Effets de bouclage}

Restent des cas difficiles à analyser, comme :

Amoureuse comme elle était, (...)

Etranger comme je le suis à tout négoce et trafic, (...)

dans lesquels le différentiel est identique au prédicat de la proposition matrice (du fait du vicaire (le) être). D'où les gloses : [étant] amoureuse comme elle était [amoureuse] ; [étant] étranger à tout négoce et trafic comme je suis [étranger à tout négoce et trafic]. Ces structures peuvent s'analyser comme des effets de bouclage, avec identité entre le paramètre et le différentiel, produisant un effet de haut degré : voir C. Fuchs et P. Le Goffic (2005). Cette structure peut se complexifier par l'introduction d'une modalité de phrase dans la subordonnée, ainsi :

Méfiant comme elle te connaît, (...)

se glosera par : [étant] méfiant comme elle te connaît [être méfiant = comme elle connaît que tu es méfiant].

\subsection{En bref}

L'examen de la subordonnée permet donc d'épingler le constituant qui est 'différentiel' par rapport à la proposition matrice et de lui attribuer une catégorie (entité, qualité ...) ainsi qu'un rôle (actant, circonstant, ...) sémantiques. Cependant identifier le différentiel ne préjuge pas du rôle qui est le sien dans la comparaison; en particulier le différentiel n'est pas nécessairement le comparant (ou repère). Certes, dans les exemples prototypiques, où la comparaison opère entre deux entités, du type :

\section{Jean est plus grand que son frère}

le différentiel (son frère) est aussi le comparant et son constituant parallèle dans la matrice (Jean) est le comparé. Mais c'est loin d'être toujours le cas. Un différentiel-entité peut n'être pas le comparant mais une variable du paramètre, ainsi dans : 
où l'on ne compare pas le chien et le chat de Jean, mais l'amour de Jean pour l'un et l'autre. Quant à un différentiel-qualité, il peut être aussi bien l'équivalent du paramètre qu'une circonstance :

\section{Jean est plus brillant qu'intelligent \\ Jean est plus brillant maintenant que jeune (= « dans sa jeunesse »).}

Ceci montre qu'il est nécessaire, et pour spécifier le rôle sémantique du différentiel dans la subordonnée et pour identifier les termes comparés dans la structure comparative, de passer par la restitution de la prédication complète de la subordonnée et l'appariement du différentiel avec un constituant qui lui est parallèle dans la prpoosition matrice ; c'est ce que nous allons examiner à présent.

\section{Le type de constituant parallèle et la correspondance matrice/subordonnée}

Une fois identifié le différentiel, il faut pouvoir retrouver le (ou les) constituant(s) de la proposition matrice A parallèle(s) au(x) constituant(s) différentiel(s) de la subordonnée $\mathrm{B}$, de façon à identifier les termes entre lesquels joue la comparaison. Cela passe par la restitution de la prédication complète de $\mathrm{B}$, qui suppose, d'une part la restitution des éléments de $\mathrm{A}$ représentés par des anaphores ou ellipsés en $\mathrm{B}$, d'autre part, la restitution des schémas prédicatifs ou propositionnels de $\mathrm{A}$ induits par la présence d'un verbe modal en $\mathrm{B}$. Comme on le verra, la difficulté d'identification du constituant parallèle tient en particulier à la question des « niveaux » où joue la correspondance entre $\mathrm{A}$ et $\mathrm{B}$, et des ambiguïtés afférentes.

\subsection{La restitution des anaphores}

Seules sont pertinentes pour l'analyse des comparatives les anaphores qui opèrent au niveau du prédicat de la proposition matrice, càd. du paramètre. On comparera ainsi :

Marie est plus jolie que ne l'est sa sœur/que n'est sa sœur/qu'est sa sœur/que sa sœur sæur

Marie travaille plus que ne le fait sa sœur/que ne fait sa sœur/que fait sa sæur/que sa

Les anaphores de constituants ne sont pas caractéristiques des comparatives, comme par exemple :

Souvent un jeune homme quitte sa promise pour une jeune fille plus riche qu'elle ('elle' = 'sa promise').

L'anaphore du prédicat se fait par les vicaires être (avec un prédicat attributif) ou faire (avec un prédicat plein). L'anaphore peut être assurée, soit par l'ensemble pronom + vicaire (le + être ; le + faire $)$ :

\footnotetext{
${ }^{5}$ Nous n'examinerons pas ici le problème du ne explétif dans la comparative.
} 
Dans cent ans l'homme sera-t-il moins malheureux qu'il l'est aujourd'hui (= qu'il est malheureux aujourd'hui)

Elle l'intimidait plus que ne l'eût fait un article de Paris (= que ne l'eût intimidé un article de Paris)

soit par le seul vicaire :

J'imagine cette ville beaucoup plus sombre qu'elle n'est (= qu'elle n'est sombre)

Elles se ressemblaient beaucoup plus que ne font d'ordinaire deux cousines (= que ne se ressemblent d'ordinaire deux cousines) Shakespeare)

Je saluai mon vieux maître comme j'eusse fait Shakespeare (= comme j'eusse salué

Les anaphores posent le problème de l'empan du prédicat anaphorisé. Il est parfois difficile de savoir si l'anaphore reprend le prédicat seul (càd. le verbe et ses arguments) ou le prédicat avec l'ensemble de ses déterminations en particulier circonstancielles. Ainsi dans :

\section{J'en parlerai ailleurs, et plus amplement et moins sobrement que je ne fais ici}

on peut hésiter à restituer 'que je n'en parle' ou 'que je n'en parle amplement et sobrement' (voir N. Fournier et C. Fuchs sous presse a).

L'anaphore du prédicat présente sur l'ellipse plusieurs avantages. D'une part, l'anaphore permet d'actualiser le prédicat de la subordonnée, et de lui donner des déterminations temporelles différentes de celles du prédicat de la proposition matrice :

C'est bien moins convaincant que ne le sera l'Ode de Claudel

Marie est plus jolie qu'elle ne l'était.

D'autre part, l'anaphore permet d'apparier très nettement autour du prédicat, plein ou vicaire, le différentiel (dans la subordonnée) et son constituant parallèle (dans la proposition matrice). Ainsi dans les exemples précédents appariera-t-on des entités de même statut actanciel, agent (instrument) : elle/un article de Paris, siège du procès : elles/deux cousines ou patient : mon vieux maître/Shakespeare, ou des circonstances : dans cent ans/aujourd'hui.

L'anaphore permet en particulier de lever les ambiguités et d'assigner au différentiel un statut actanciel distinctif. Ainsi le vicaire dans :

La reine sa mère ne l'aimait pas plus tendrement que ne faisait Anne d'Espagne

impose-t-il de lire le différentiel Anne d'Espagne comme le siège du procès ('aimer', vicarisé par faisait), alors que l'ellipse :

La reine sa mère ne l'aimait pas plus tendrement qu'Anne d'Espagne

donnerait un énoncé ambigu, le différentiel pouvant être le siège ('qu'Anne d'Espagne ne l'aimait') ou le patient ('qu'elle n'aimait Anne d'Espagne') du procès.

Enfin, l'anaphore peut servir à déterminer le niveau du prédicat ellipsé, en particulier dans le cas de prédication seconde. Ainsi dans un exemple comme :

Marie a engagé un collaborateur plus efficace que Jean 
le choix du vicaire peut permettre de spécifier le prédicat ellipsé. On opposera ainsi que ne l'est Jean (le prédicat ellipsé est 'être efficace', prédicat second) à que ne l'a fait Jean (le prédicat ellipsé est alors 'engager un collaborateur efficace', prédicat primaire de la proposition matrice). L'anaphore permet de spécifier du même coup quel est le constituant parallèle au différentiel Jean (un collaborateur ou Marie).

L'exemple de Fénelon cité plus haut :

Les méchants princes souffraient dans le Tartare des supplices infiniment plus rigoureux que (...)

va dans le même sens. Le choix du vicaire pourrait servir à l'identification du prédicat ellipsé, en contrastant que ne le sont les supplices ordinaires/que ne le faisaient les autres coupables d'une condition privée. Ceci ne vaut évidemment que si les deux prédicats (second et primaire) sont contrastifs (l'un de type 'faire', l'autre de type 'être'). L'anaphore n'est plus distinctive dans le cas de deux prédicats de même type sémantique ; ainsi dans :

Jacques est sensible à des êtres plus doux que (ne l'est) sa sœur

la vicarisation se fait par être pour les deux prédicats 'être sensible' et 'être doux', en sorte qu'il y a ambiguiité sur le niveau du prédicat ellipsé.

Les limites de l'anaphore tiennent aux restrictions (syntaxiques et sémantiques) qui pèsent, en français moderne, sur le vicaire faire, qui a perdu la capacité à vicariser un procès non agentif et à construire un complément direct. Ainsi les exemples suivants sont-il sentis comme archaïques ou littéraires :

bonne

(ce que) je ne pouvais prendre avec autant de philosophie que faisait mon ancienne

Elles se ressemblaient beaucoup plus que ne font d'ordinaire deux cousines (avec un verbe non agentif) direct).

Je saluai mon vieux maître comme j'eusse fait Shakespeare (avec un verbe transitif

Dans ce cas, l'usage standard est la construction prépositionnelle du complément : Je saluai mon vieux maître comme j'eusse fait pour Shakespeare ou la répétition du verbe de la matrice : comme j'eusse salué Shakespeare (voir N. Fournier et C. Fuchs 1999).

\subsection{La restitution des ellipses}

Nous ne rentrerons pas ici dans le détail des ellipses (nous renvoyons à N. Fournier et C. Fuchs sous presse a). Nous nous contenterons de rappeler que l'ellipse implique une restitution notionnelle du prédicat, qui reste largement sous-déterminée, en particulier au niveau des marques de temps et de modalité (ce que nous noterons par la forme indéterminée de l'infinitif).

La restitution de la relation prédicative de la subordonnée prend appui d'une part sur la catégorie sémantique du différentiel, d'autre part sur la relation prédicative de la proposition matrice, qui est énoncée avant la subordonnée (les subordonnées à portée intraprédicative ne sont jamais antéposées) et qui est en mémoire pour l'interprétation de la subordonnée.

L'interprétation de la structure comparative repose sur un processus complexe, dont on peut distinguer (métalinguistiquement) les composantes suivantes : l'appariement du différentiel avec un constituant parallèle dans la proposition matrice (sur la base de propriétés 
congruentes), la restitution des ellipses, càd. la copie dans la subordonnée de tout ou partie du prédicat de la proposition matrice (à l'exclusion du constituant parallèle), l'interprétation du différentiel par rapport à la relation prédicative restituée.

\subsubsection{Le différentiel est une entité}

Deux cas sont alors possibles :

a) Le différentiel peut s'apparier avec le premier actant du prédicat (le support de la relation prédicative), et la restitution affecte le prédicat complet de la proposition matrice, qui est copié dans la subordonnée : ses pieds']

Ce garçon n'était guère plus solide sur ses pieds que son compagnon ['être solide sur

(...) un garçon qui avait autant voyagé que Robinson ['voyager'] ;

Marie achète plus de livres que sa sæur ['acheter des livres']

Marie part plus souvent en vacances que sa sour ['partir en vacances'].

b) Quand le prédicat est assorti d'une variable (argument, circonstance), le différentiel-entité peut s'apparier, non avec le sujet mais avec la variable. La restitution affecte alors le prédicat (moins la variable) et son support sujet :

Il préfère étaler son intelligence que ['il aimer étaler'] ses dons

Elle sert aussi bien à la défense que ['elle servir'] à l'attaque.

Les ambiguïtés sont liées aux cas où le différentiel-entité peut s'apparier à deux constituants parallèles contrastés sur le plan actanciel, ce qui entraîne deux restitutions possibles des ellipses :

Vous saurez la soigner autrement mieux que ['vous soigner'] ma mère / que ma mère ['la soigner'].

\subsubsection{Le différentiel est une qualité}

Il s'apparie avec la qualité correspondante dans la matrice, qui constitue le prédicat ou une variable du prédicat :

Cette situation est moins angoissante qu'absurde

Il m'a répondu aussi efficacement qu'aimablement

Il joue du piano avec plus de conviction que de talent.

La restitution affecte alors soit la copule et le sujet (que ['cette situation être'] absurde), soit le prédicat (sans sa variable) et le sujet (que ['il me répondre'] aimablement; que ['il jouer du piano'] (avec du) talent).

\subsubsection{Le différentiel est une circonstance}

Il s'apparie avec la circonstance correspondante dans la proposition matrice et c'est l'ensemble de la relation prédicative qui est restituée dans la subordonnée :

Le printemps en Bretagne est plus doux que ['le printemps être doux'] aux environs de

Il est plus grand mort que ['il être grand'] vivant 
Aujourd'hui, nous nous précipitons dans le palais avec encore plus d'empressement que ['nous se précipiter dans le palais avec empressement'] autrefois.

On notera que le constituant parallèle peut être un constituant zéro (marquant une localisation par défaut) :

Je lui ai répondu ['cette fois-là'] aussi tranquillement que la première fois

Je travaille mieux ['ici'] qu'à la campagne.

\subsubsection{En bref}

Les comparaisons en que apparaissent ainsi comme des structures nettement balisées par l'association du déclencheur et de que, qui permettent de varier l'appariement entre différentiel et constituant parallèle et de contraster (par la restitution de l'ellipse) soit deux prédicats différents, soit le même prédicat assorti d'une variable.

En revanche, les comparaisons en comme ne permettent pas cette mise en balance des constituants et les variations entre différentiel et constituant parallèle. Ce sont en effet des comparaisons qui qualifient directement le prédicat et contrastent des manières de faire ou d'être, plutôt que les actants engagés dans le prédicat. Il apparaît ainsi qu'avec comme, le contraste entre des entités autres que le sujet fait sortir de la comparaison pour des constructions prédicatives (considérer comme) ou des valeurs de type "coordination" (Il aime le jazz comme l'opéra), ou bien va de pair avec une portée extraprédicative : Je m'entretenais avec elle, comme avec un être réel. Par ailleurs, on ne peut pas contraster des qualités : ??? Cette situation est angoissante comme absurde; ??? Il m'a répondu aimablement comme efficacement, et le contraste sur les circonstances tire vers une portée extraprédicative : Je me suis rencontré entre les deux siècles comme au confluent de deux fleuves. Voir infra, $\S 5$.

\subsection{La restitution des schémas induits par une modalité de prédicat ou de phrase}

Quand le verbe de la subordonnée est un verbe modal, le prédicat ou la phrase sur quoi il porte est anaphorisé par le:

Elle ne s'effraya pas autant qu'elle l'aurait dî (= qu'elle aurait dû s'effrayer)

La mer était plus impressionnante que ne l'imaginait l'enfant (= que l'enfant n'imaginait qu'elle était impressionnante)

ou par une anaphore zéro :

N'ai-je pas fait plus que je ne devais (= que je ne devais faire)

Je suis plus ferme que vous ne pensez (= que vous ne pensez que je suis ferme).

Dans ce cas, face au différentiel de modalité, on peut postuler au titre de constituant parallèle une modalité par défaut (de prédicat ou de phrase) dans la matrice : Elle ne s'effraya pas ['dans les faits'] autant que ... ; ['il est de fait que'] la mer était plus impressionnante que ...

4.4. Les niveaux de la correspondance proposition matrice/subordonnée

L'exemple de Fénelon :

Les méchants princes souffraient dans le Tartare des supplices infiniment plus rigoureux que les autres coupables d'une condition privée 
a montré que le différentiel les autres coupables d'une condition privée avait pour constituant parallèle non pas des supplices, support de la prédication seconde (sur laquelle porte le déclencheur plus) mais les méchants princes, support de la prédication primaire de la matrice et que la restitution de l'ellipse devait remonter jusqu' au niveau du prédicat primaire 'souffrir dans le Tartare des supplices rigoureux'. Ceci illustre un problème plus général qui est celui des « niveaux » auxquels opère la comparaison et de l'empan du paramètre (et corollairement de la restitution de l'ellipse dans la subordonnée). L'exemple de Fénelon illustre ainsi un cas de « remontée » jusqu'au prédicat primaire, que l'on peut contraster avec une prédication de même niveau, engageant des constituants co-planaires - qu'il s'agisse d'entités : des supplices infiniment plus rigoureux que les supplices ordinaires, ou de qualités : des supplices infiniment plus rigoureux que spectaculaires.

Dans les comparaisons de même niveau, du type :

Marie est plus jolie que sa cousine/que jamais/qu'elle n'était/qu'en Bretonne/que belle

tous les constituants parallèles possibles sont co-planaires, càd. qu'ils sont au même niveau relativement au prédicat 'être jolie'. Selon les cas, on compare deux entités (Marie/sa cousine) au regard du prédicat 'être jolie', ou bien une entité (Marie) à elle-même au regard du même prédicat assorti d'une variable, en l'occurrence une circonstance ('être jolie maintenant'/'être jolie jamais' ou 'avant' ; 'être jolie au naturel'/'être jolie en Bretonne'), ou au regard de deux prédicats ('être jolie'/'être belle').

Ainsi dans l' exemple :

Les laides qui plaisent allument de plus furieuses passions et de plus violents engouements chez les hommes que les plus parfaites beautés de la terre

le différentiel les plus parfaites beautés de la terre est-il apparié au sujet les laides qui plaisent, et le paramètre est le prédicat global 'allumer des passions furieuses et des engouements violents chez les hommes'. Mais on pourrait avoir les combinaisons suivantes, moyennant des variations sur le paramètre : Les laides qui plaisent allument de plus furieuses passions chez les hommes que chez les femmes/que des passions tièdes/que des sentiments d'estime/qu'elles ne les éteignent, avec appariement du différentiel avec une circonstance (chez les hommes/chez les femmes), une qualité (tièdes, furieuses), une entité abstraite (des sentiments d'estime/de furieuses passions), un procès (allumer de furieuses passions/les éteindre) ou, comme c'est le cas dans l'exemple attesté, avec le sujet.

Les remontées sont donc liées aux cas de prédication seconde (comme dans l'exemple de Fénelon, cité plus haut), dans lesquels le prédicat est assorti d'une variable. Empan étroit, quand on reste au niveau de la prédication seconde :

Marie a une plus voiture plus rapide qu'une Ferrari (le prédicat est 'être rapide')

ou empan large, quand on remonte au niveau de la prédication primaire : rapide').

Marie a une voiture plus rapide que son frère (le prédicat est 'avoir une voiture

Les remontées ne sont pas possibles avec tous les types d'enchâssement. Elles sont impossibles avec des complétives enchâssées ; dans : 
la comparaison s'arrête au niveau du prédicat enchâssé : Jacques pense que Paul est plus compétent que Jean [n'est compétent] et ne peut pas remonter jusqu'au verbe d'attitude propositionnelle ; l'énoncé ne peut pas, en effet, signifier : * Jacques pense que Paul est plus compétent que Jean [ne le pense].

Quant à ce qu'on pourrait appeler les « descentes », elles correspondent à des exemples dans lesquels le paramètre est assorti d'une variable, comme dans :

\section{Marie est plus sensible à la peinture qu'à la musique}

(vs. Marie est plus sensible à la peinture que sa sæur).

Ce qui est comparé c'est une entité (Marie) relativement à un même paramètre ('être sensible') assorti de deux variables contrastives ('à la musique'/'à la peinture').

\subsection{En bref}

La restitution des anaphores et des ellipses permet donc d'apparier le différentiel de la subordonnée avec un constituant parallèle de la proposition matrice et de restituer le prédicat commun à la matrice et à la subordonnée. Les différents problèmes rencontrés font apparaître, d'une part la différence fondamentale entre les comparatives en que qui se prêtent à l'appariement de constituants parallèles et les comparatives en comme qui s'y prêtent mal, d'autre part le fait que les difficultés à apparier les constituants, et les ambiguïté qui en résultent, tiennent au niveau auquel joue la restitution du prédicat et aux différences d'empan dans sa restitution.

\section{Le type de termes comparés}

L'analyse fait apparaître que si, dans les cas prototypiques (Jean est plus grand que Paul), la construction quantitative consiste à comparer deux entités ou objets du monde (Jean et Paul), en revanche, dans nombre de cas, l'identification des termes comparés pose problème. En outre, les comparaisons qualitatives en comme ont un fonctionnement très différent de celui des comparaisons quantitatives en que, dans la mesure où elles n'instaurent qu'indirectement une comparaison entre deux termes, par ailleurs dissymétriques.

\subsection{Les termes comparés quantitativement}

Du point de vue sémantique, le schéma de base est celui où deux entités sont comparées relativement à un prédicat identifié comme étant le paramètre ${ }^{6}$. Dans les cas problématiques, le choix de l'empan du paramètre se fait, on vient de le voir, à partir des indices livrés par le différentiel et son constituant parallèle. Le schéma de base se complexifie lorsque les deux entités sont comparées relativement à un prédicat assorti d'une variable, ou à deux prédicats. Enfin, lorsque la construction ne concerne qu'une seule entité face à deux prédicats ou à un

\footnotetext{
${ }^{6}$ Nous ne prenons pas ici en compte le cas des subordonnées à portée extraprédicative, où les termes comparés sont les deux situations (présentées comme analogues) auxquelles correspondent les deux propositions (Il a remboursé ses dettes, comme/ainsi que son frère l'avait fait avant lui), ni celui des subordonnées à portée exophrastiques, où les termes comparés sont les deux énonciations (Comme disent les braves gens, (je dis) c'est un cassement de tête).
} 
prédicat assorti d'une variable l'identification des termes comparés devient plus problématique.

\subsubsection{Deux entités relativement à un prédicat}

Dans le cas prototypique, deux entités singulières spécifiques, renvoyant préférentiellement à de l'humain, sont comparées quant au degré auquel elles vérifient chacune le même prédicat (qui coïncide avec le paramètre). Ainsi :

\section{Jeanne est plus belle que Marie}

où la comparaison entre les deux entités 'Jeanne' et 'Marie' revient, pour ainsi dire, à mettre dans chacun des deux plateaux de la balance, leur 'beauté' respective pour la « soupeser ». La comparaison est facilitée par l'appartenance des deux entités à une même classe : classe des 'humains féminins' dans cet exemple, ou classe des 'voitures' dans :

La voiture de Jeanne est moins rapide que celle de Marie.

Un cas particulier est celui de la comparaison à parangon, où une entité spécifique est comparée à une entité générique réputée vérifier à son plus haut degré le prédicat :

Sa toison était plus blanche que neige

Il est aussi aimable qu'une porte de prison.

On remarquera néanmoins que la comparaison à parangon prototypique est construite avec comme (plutôt qu'avec 'déclencheur $+q u e$ '), du fait précisément de cette dissymétrie entre les deux types d'entités comparées.

Les termes comparés renvoient préférentiellement à des entités définies, mais d'autres cas se rencontrent. Ainsi dans la question :

\section{Quoi de plus espagnol que Cervantès?}

(càd. 'y a-t-il quelque chose qui soit plus espagnol que Cervantès ?'), le terme indéfini quoi est comparé au représentant prototypique de 'l'hispanité'.

Les termes comparés ne renvoient pas non plus nécessairement à des entités singulières correspondant à des êtres humains ou à des objets concrets du monde. Ils peuvent aussi renvoyer à des entités notionnelles, abstraites. Ainsi, dans :

\section{Etre rayé de dessus l'Etat me serait moins fâcheux qu'être effacé de votre mémoire}

sont comparés '(le fait d')être rayé de dessus l'Etat' et '(le fait d')être effacé de votre mémoire' quant au degré de leur caractère 'fâcheux' - la quasi-synonymie entre les deux verbes conduisant, en définitive, à mettre en parallèle 'l'Etat' et 'vous'.

\subsubsection{Deux entités relativement à un prédicat assorti d'une variable $X$}

Lorsque le prédicat (au regard duquel les deux entités sont comparées) est assorti d'une variable (mise en évidence par le différentiel), cela revient, d'une certaine façon, à le dédoubler.

La variable en question peut correspondre :

a) à l'objet du verbe : 
Marie aime plus son chien que Jeanne son chat

compare les deux entités 'Marie' et 'Jeanne' quant au degré de leur 'amour' pour $\mathrm{X}=$ 'leur animal domestique respectif' ;

b) au complément de l'adjectif :

Aristote n'est pas moins essentiel à toute métaphysique que ne sont les éléments d'Euclide à la géométrie

compare les deux entités 'Aristote' (ou plutôt ses œuvres) et 'Euclide' (ou plutôt ses éléments de mathématiques) quant à leur degré respectif $d$ ' "essentialité' relativement à $X=$ 'la métaphysique' vs. 'la géométrie' ;

c) à un circonstant, temporel, spatial et/ou notionnel :

Marie est plus belle aujourd'hui que Jeanne hier

(...) la vieille Europe où l'homme s'est montré bien plus destructeur qu'ici la nature

Vous êtes beaucoup plus heureux en votre race que Pompée n'a été en la sienne.

Dans tous ces cas, les termes comparés sont bien les deux entités supports du prédicat ('Marie' et 'Jeanne', 'l'homme' et 'la nature', 'vous' et 'Pompée'), considérées quant au degré où elles vérifient un même prédicat considéré sous $\mathrm{X}=$ deux angles différents.

Cette configuration est parfois utilisée dans le cas d'une comparaison à parangon :

Nous connaissons nos signaux mieux qu'un prêtre son bréviaire.

\subsubsection{Deux entités relativement à deux prédicats}

Le dédoublement de point de vue du côté prédicatif peut aussi aller jusqu'à une totale différenciation au niveau même du lexème verbal. Nombreux sont les cas de constructions symétriques entre la proposition matrice et la subordonnée, où deux entités sont comparées relativement à deux prédicats différents. Exemples :

Ma vie était maintenant aussi sotte et ennuyée que la sienne douloureuse La nuit fut aussi douce, aussi calme, que la journée avait été chaude et animée.

Dans des exemples de ce type, les deux entités qui constituent les termes comparés sont de la même classe ('ma vie', 'la sienne') ou contrastent au sein d'une même classe superordonnée ('la nuit', 'la journée'), et les deux prédicats sont tantôt quasi-synonymes ou sémantiquement proches ('être sotte et ennuyée', 'être douloureuse') tantôt antinomiques ('être douce et calme', être chaude et animée'). Les deux entités sont comparées quant au degré où elles vérifient respectivement l'un ou l'autre des deux prédicats sémantiquement apparentés. On pourrait ici considérer ces deux prédicats comme deux instances différentes d'un même domaine prédicatif superordonné ('sottise et ennui' et 'douleur' constituant deux instances d'un domaine d' 'émotions négatives', 'douceur et calme' et 'chaleur et animation' deux instances opposées d'un domaine d' 'impressions').

Rares sont les exemples où les contenus semblent n'avoir guère de rapport sémantique, tel celui-ci : 
J'ai vu mourir presque autant de lois scientifiques que Poincaré a promené de discours sur des tombes de soldats inconnus

Quel rapport entre le prédicat 'voir mourir des lois scientifiques' et le prédicat 'promener des discours sur des tombes de soldats inconnus', hormis la seule notion de mesure quantitative élevée qu'implique le second lorsqu'il est prédiqué d'un homme politique ? Cette absence de lien sémantique rend plus difficile la reconstitution d'une parenté de points de vue selon lesquels les deux entités 'je' et 'Poincaré' seraient comparées. En définitive, l'énoncé semble plutôt vouloir insister sur 'le très grand nombre de lois scientifiques que j' ai vues mourir', en recourant à une structure comparative qui transpose au plan situationnel une comparaison à parangon.

\subsubsection{Une entité relativement à deux prédicats}

Plus loin encore des constructions prototypiques sont les cas où une seule entité est en jeu.

Dans ces cas, le dédoublement s'opère nécessairement du côté prédicatif. Il peut s'agir de deux prédicats entièrement distincts :

\section{Les petites Irlandaises dansaient mieux qu'elles ne chantaient \\ Le prince fut aussi vite calmé qu'il avait été irrésistiblement enivré.}

Ici, l'unique entité ('les petites Irlandaise' ; 'le prince') est considérée quant au degré respectif où elle vérifie chacun des deux prédicats ('bien danser' vs. 'bien chanter' ''être vite calmé' $v s$. 'être vite enivré'). Peut-on dire alors que cette unité est comparée à elle-même ? En présence d'une seule entité, on est plutôt tenté de chercher dans d'autres constituants, càd. du côté prédicatif, la dualité 'comparé/comparant' : on dira facilement, par exemple, que sont comparées quantitativement 'la qualité de danse des petites Irlandaises' et 'leur qualité de chant', ou bien 'la vitesse de retour au calme du prince' et 'la vitesse de son irrésistible enivrement'.

A fortiori, dans :

\section{Le vieil Ennius disait déjà qu'il avait autant d'âmes qu'il avait appris de parlers}

le récepteur verra-t-il facilement une comparaison quantitative entre le nombre 'd'âmes (possédées par le vieil E.)' et le nombre 'de parlers (qu'il avait appris)'. De même, dans :

\section{Les hommes risquent davantage en vous laissant vivre qu'en vous attaquant}

on aura tendance à restituer une comparaison entre deux états de choses ('vous laisser vivre' et 'vous attaquer') relativement au degré respectif de 'risque' qu'ils impliquent pour 'les hommes'. En d'autres termes, dès lors que la construction ne présente plus qu'une seule entité en position de support du (ou des) prédicats(s), on est tenté de ne plus assimiler cette entité à un terme comparé et de rechercher du côté prédicatif deux éléments susceptibles d'être mis en parallèle.

\subsubsection{Une entité relativement à un prédicat assorti d'une variable $X$}

Le dédoublement du côté prédicatif peut se faire simplement en assortissant le prédicat d'une variable.

Cette variable peut être une modalité. Modalité de phrase, comme dans : 

belle)

Elle est plus belle que je ne croyais (= elle est plus belle que je ne croyais qu'elle était

ou modalité de prédicat, comme dans :

Elle travaille autant qu'elle le peut (= elle travaille autant qu'elle peut travailler).

Dans le premier exemple, l'entité 'elle' (relativement à son degré de beauté effective) est contrastée avec la même entité 'elle' (relativement à son degré de beauté imaginée par moi) - autrement dit on la considère relativement à son degré de beauté sous $\mathrm{X}=$ dans deux univers de croyance différents. Dans le second exemple, l'entité 'elle' (relativement à son degré de travail effectif) est contrastée avec la même entité 'elle' (relativement à son degré de capacité de travail) - on la considère donc relativement à son degré de travail sous $\mathrm{X}=$ selon les deux modalités de l'effet et de la puissance. La question se pose, ici encore, de savoir si l'on considère que l'unique entité est comparée à elle-même (quant au degré ...) ou bien si l'on compare quantitativement 'sa beauté réelle' à 'sa beauté imaginée' ou 'son travail effectif' et 'sa capacité de travail'.

La variable peut également être une circonstance. Ainsi dans :

La vie était alors plus agréable qu'aujourd'hui

Il paraît encore plus grand mort que vivant

où une entité ('la vie', 'lui') fonctionne comme support d'un prédicat ('être agréable', 'être grand') assorti de variables contrastées $\mathrm{X}$ ('alors $\boldsymbol{v} \boldsymbol{s}$. aujourd'hui', 'mort $\boldsymbol{v s}$. vivant'). Ici encore, on voit que le contraste induit sur le prédicat par la variable conduit à rechercher des éléments de comparaison du côté prédicatif: 'la vie d'alors' par rapport à 'la vie d'aujourd'hui' ou 'lui mort' par rapport à 'lui vivant'.

Lorsque le côté prédicatif comporte des éléments susceptibles d'être compris comme des entités à part entière, ces éléments sont d'autant plus facilement identifiés à des termes comparés. Ainsi :

Votre article sur Volupté, mon cher Hippolyte, a rencontré autant d'admirateurs que de lecteurs

se laisse-t-il interpréter comme comparant le nombre ' $d$ 'admirateurs (de l'article en question)' et son nombre 'de lecteurs'. De même :

Un modèle est plus clairement exprimé par le pinceau abondant et facile d'un coloriste que par le crayon d'un dessinateur

sera-t-il compris comme comparant 'le pinceau du coloriste' et 'le crayon du dessinateur' quant au degré relatif de 'clarté d'expression du modèle (qu'ils permettent)'.

\subsection{Les termes comparés qualitativement}

Contrairement aux comparaisons quantitatives construites par des corrélatives en que, les comparaisons qualitatives en comme ${ }^{7}$ n'instaurent qu'indirectement la mise en parallèle de

\footnotetext{
${ }^{7}$ Nous ne développerons pas ici les corrélatives qualitatives en tel que, même que, autre que.
} 
deux termes comparés. En effet, l'apport sémantique propre de comme est de construire une identification sur la manière (d'être ou de faire).

Dans les cas prototypiques, la manière de vérifier le prédicat en $\mathrm{A}$ est identifiée à la manière de vérifier ce même prédicat en $B$. Ainsi, dans :

\section{Chacun mangeait comme on mange en province}

Il aimait Célestin comme on aime un vieil ami de quarante ans

il ne s'agit pas de comparer deux entités ('chacun' et 'on', ou 'il' et 'on'), mais de qualifier la manière de 'manger' (de chacun) ou sa manière à lui d' 'aimer Célestin' en l'identifiant à la manière habituelle de 'manger' (en province) ou à la manière habituelle d' 'aimer un vieil ami de quarante ans'. On s'explique dès lors que nombre de comparaisons de ce type comportent des arguments et des prédicats génériques en $B$, représentant en quelque sorte une manière «de référence», ou une manière caractéristique de vérifier le prédicat: ainsi dans la comparaison à parangon :

\section{Elle m'a traité comme un chien}

càd. 'elle m'a traité de la manière caractéristique dont on traite un chien (en l'occurrence de la manière la plus désagréable qui soit)'. Pour autant, la manière de référence reste indéterminée :

\section{Jean s'en alla comme il était venu}

signifie 'Jean s'en alla de la manière (quelle qu'elle soit) dont il était venu', mais rien ne spécifie cette manière : était-ce discrètement et en silence, ostensiblement et bruyamment, ... ? La manière n'est précise que dans la mesure où le récepteur peut l'inférer de ses propres connaissances de la situation, ou de ses connaissances d'univers :

\section{Il ment comme il respire}

se comprend comme 'il ment de la manière dont il respire, càd. le plus naturellement du monde et/ou sans cesse'. De même :

Une démocratie imposée par la force ressemble à la dictature comme une goutte d'eau à une autre goutte d'eau

permet de comprendre que le type de démocratie en question ressemble 'absolument/totalement' à la dictature (au point qu'on ne puisse les distinguer), puisque c'est là la manière dont deux gouttes d'eau se ressemblent.

On s'explique que, lorsque le prédicat de A n'est pas le même que celui de B, les domaines sémantiques auxquels renvoient ces prédicats puissent être beaucoup plus éloignés les uns des autres que dans le cas des comparaisons quantitatives en que. Devant un exemple tel que :

\section{Schumann}

Il $m$ 'arrive de taper un rapport affreusement ennuyeux comme un autre jouerait du

le récepteur peut se demander à quelle manière de 'jouer du Schumann' l'énoncé fait référence - l'interprétation la plus plausible étant 'il m'arrive (même) de taper un rapport 
affreusement ennuyeux avec plaisir' ; mais l'idée d'une comparaison entre 'moi' et 'un autre' ne lui viendra sans doute pas.

A fortiori s'agissant de la pure manière d'être (sans autre spécification), le rôle caractérisant de comme s'éloigne d'une comparaison entre deux entités distinctes. Les énoncés :

C'est plein de livres comme tu les aimes

Vous imaginez-vous, Monsieur de Pourceaugnac, qu'une fille comme la mienne soit aussi affamée de mari?

ne comparent pas deux occurrences différentes de 'livres' ou 'filles', mais qualifient un type de livre ou un type de fille.

On voit que les comparaisons qualitatives en comme n'ont pas pour objectif premier de comparer deux entités relativement à un paramètre. Résultativement, pourtant, elles peuvent avoir cet effet. Le cas le plus net est celui où la manière d'être (renvoyant à une qualité gradable) d'une entité de $\mathrm{A}$ est identifiée à celle d'une entité (le plus souvent générique) de $\mathrm{B}$; exemple :

Hassan était donc nu, - mais nu comme la main (= aussi nu que la main, càd. 'complètement nu').

Ainsi que nous l'avons vu au $\S 2.1 .1$, le glissement de la qualification à la quantification procède alors du caractère gradable de la qualité ('nu'). Dans un cas de ce type, l'entité 'Hassan' est, résultativement, comparée à l'entité 'la main', en tant que partageant la même manière d' 'être nu' (càd. ici le même haut degré de 'nudité'). Il en va de même pour toutes les comparaisons à parangon, du genre bête comme ses pieds, aimable comme une porte de prison, gai comme un pinson, joli comme un cœur,... On remarquera la dissymétrie entre les deux entités comparées : en $\mathrm{A}$, une entité spécifique en $\mathrm{A}$, et en $\mathrm{B}$ une entité générique, qui constitue le représentant prototypique de la classe des entités possédant la propriété en question : c'est le «modèle » par excellence, celui qui vérifie le prédicat au plus haut degré. Contrairement à ce qui se passe avec que, on voit qu'il n'y a pas de parallélisme entre les deux termes comparés.

Lorsque la gradabilité du prédicat n'est pas en jeu, il arrive que, la structure s'y prêtant, on puisse trouver deux entités entre lesquelles une comparaison est établie à travers l'identité de manière sur le prédicat. Ainsi :

Qu'avez-vous à me regarder comme une oie ? (= qu'avez-vous à me regarder à la manière d'une oie) ${ }^{8}$

instaure par là même une sorte de comparaison entre 'vous' et 'une oie'. Dans le cas de structures symétriques avec variation du sujet et de l'objet, un double parallélisme s'établit entre deux séries d'entités :

Ces beaux silences qui scellent les âmes comme un ciment les pierres d'une arche

\footnotetext{
${ }^{8}$ L'énoncé est structurellement ambigu entre la fonction sujet et la fonction objet du terme 'oie' : 'comme une oie regarde'/ 'comme on regarde une oie'; il semble pourtant que la première solution soit la plus plausible dans le contexte : - Et bien, quoi ? Servez le champagne, dit-elle. Qu'avez-vous à me regarder comme une oie? (Zola).
} 
(les 'beaux silences' sont aux 'âmes' ce que 'un ciment' est aux 'pierres d'une arche'). De même :

\section{Pour que je la console comme un frère aîné une petite sæur sans amant}

Pour autant, le fait de pouvoir reconstruire des termes comparés apparaît comme une sorte d'effet en bout de chaîne et, contrairement aux termes comparés avec que (qui sont sémantiquement co-planaires) ceux que comme permet de comparer se révèlent être sémantiquement dissymétriques.

\section{Le type de comparaison et les effets pragmatiques}

A partir de la nature du déclencheur (et de son éventuel modifieur) et/ou de la nature de la 'cheville' (que ou comme), ainsi que des types de paramètres et de termes comparés, on peut identifier au sein des deux grands types de comparaison (quantitative/qualitative), les configurations prototypiques ainsi que divers cas particuliers induisant certains effets pragmatiques spécifiques.

\subsection{Les comparaisons quantitatives}

A la suite de R. Rivara (1990 et 1995), nous considérons qu'il n'y a que deux types de relation de comparaison quantitative : l'égalité ou l'inégalité - cette dernière se dédoublant secondairement en 'supériorité' et 'infériorité' en fonction de l'orientation de l'échelle. Il s'agit donc d'établir une (in)égalité de degrés sur l'échelle associée à un prédicat qui renvoie à une qualité, une propriété, ou un procès en situation, à propos de deux entités distinctes ou d'une même entité considérée de deux points de vue.

Les cas particuliers induisant certains effets pragmatiques sont liés à trois principaux ordres de facteurs : l'orientation de l'échelle, l'opération de parcours et les glissements entre quantification et qualification.

\subsubsection{L'orientation de l'échelle}

L'échelle d'intensité ou de quantité associée au paramètre peut être orientée "vers le haut» (c'est l'échelle des 'grandes quantités') ou « vers le bas » (c'est l'échelle des 'petites quantités') : voir sur ce point R. Rivara (1990, chapitres 1 et 2). L'inégalité opérant sur l'échelle des grandes quantités donne ce que l'on a coutume d'appeler la 'supériorité', celle sur l'échelle inverse ce que l'on appelle l' 'infériorité'.

S'agissant d'égalité, les déclencheurs aussi et autant indiquent que l'on se situe sur l'échelle des grandes quantités (ils correspondent à 'aussi/autant beaucoup'), alors que pour construire une égalité sur l'échelle des petites quantités, il faut recourir à aussi peu. On distinguera ainsi :

Paul est aussi bavard que Jean

et Paul est aussi peu bavard que Jean

ou bien :

Paul travaille autant que Jean

et Paul travaille aussi peu que Jean. 
L'orientation de l'échelle explique les effets résultant de la combinaison de l'opérateur de négation avec une relation de comparaison. Nier une égalité sur l'échelle des grandes quantités revient à construire une inégalité de type infériorité :

Paul n'est pas aussi bavard que Jean

Paul ne travaille pas autant que Jean

équivalent à Paul est moins bavard que Jean et à Paul travaille moins que Jean. De même :

Elle ne s'effraya pas autant qu'elle l'aurait dî

équivaut à Elle s'effraya moins qu'elle (ne) l'aurait dû.

$\mathrm{Au}$ contraire, nier une égalité sur l'échelle des petites quantités (ce qui est d'ailleurs assez peu naturel) reviendrait logiquement à construire une inégalité de type supériorité :

? Paul n'est pas aussi peu bavard que Jean

? Paul ne travaille pas aussi peu que Jean

équivaudraient à Paul est plus bavard que Jean et à Paul travaille plus que Jean.

La négation d'une inégalité sur l'échelle des petites quantités (négation de l'infériorité) revient à construire une égalité sur l'échelle des grandes quantités :

Aristote n'est pas moins essentiel à toute métaphysique que ne sont les éléments d'Euclide à la géométrie

équivaut à A. est (au moins) aussi (voire même plus) essentiel que ...

Quant à la négation d'une inégalité sur l'échelle des grandes quantités (négation de la supériorité), elle peut être utilisée, comme dans l'exemple suivant, pour « renverser »une comparaison à parangon :

Le jour n'est pas plus pur que le fond de mon cœur.

En niant que 'le jour', pris ici comme parangon de la 'pureté', possède cette qualité à un degré plus élevé que 'le fond de mon cœur', l'énoncé présente ce dernier comme possédant la dite qualité au même (haut) degré (voire même plus) que le 'jour'.

\subsubsection{L'opération de parcours}

Lorsque, dans une comparaison d'inégalité, le différentiel présent en B supporte une opération de parcours, il en résulte la construction d'une valeur de 'haut degré' sur l'échelle des grandes ou des petites quantités. Le parcours peut affecter un actant (plus/moins que quiconque, plus/moins que tout), un circonstant (plus/moins que jamais) ou une modalité (plus/moins que je ne pourrais le dire, plus/moins que si...). Dans tous les cas, il s'agit d'indiquer un degré qui est supérieur (ou inférieur) à tout autre degré envisageable. L'énoncé comparatif équivaut alors à un superlatif :

Il a éprouvé mieux que personne combien nos efforts sont vains

Pauline était venue, plus maquillée et plus onduleuse que jamais 
équivalent à Il est celui qui a le plus éprouvé combien ... ; P. était venue, très maquillée et très onduleuse.

Ici encore, un différentiel pris comme parangon permet de construire le haut degré :

Elle l'aimait plus que ne l'aurait aimé une mère.

Par ailleurs, construire un degré qui dépasse tout autre degré envisageable revient au même que de construire un degré égal à tout autre degré envisageable. C'est pourquoi, avec des différentiels supportant un parcours, une comparaison d'égalité construit la même valeur de haut degré qu'une comparaison d'inégalité. Ainsi :

Elle s'éveillait aussi lucide que si elle n'avait pas fermé l'oeil (= très lucide)

Le système phonique des deux langues était aussi différent que possible (= maximalement différent)

Ce qui importerait, ce serait de définir, aussi complètement que possible, le système phonique du français au $12^{\grave{e}}$ siècle (= le plus complètement possible).

Ce qui est vrai de l'égalité sur l'échelle des grandes quantités l'est aussi de l'égalité sur l'échelle des petites quantités :

Il existe un moyen aussi peu cô̂teux que possible de réaliser une albumine iodée (= très peu coûteux, le moins coûteux possible)

On prenait dans la rue une femme, une passante, une bourgeoise, aussi peu vieille et aussi peu laide que possible (= la moins vieille et la moins laide possible).

L'articulation d'une structure comparative d'égalité (comportant un parcours) avec une autre proposition permet, on le sait, de construire des effets concessifs :

Il peut bien m'y pousser tant qu'il voudra - ça ne changera rien (= quel que soit le haut degré où il voudra me pousser, (il n'en reste pas moins que) ça ne changera rien).

\subsubsection{Glissements entre quantification et qualification}

On remarque que les comparatives quantitatives d'inégalité ou d'égalité construisant un effet de haut degré admettent une paraphrase en comme :

Il a éprouvé comme personne combien ...

Pauline était venue, maquillée et onduleuse comme jamais

Elle l'aimait comme l'aurait aimé une mère

Elle s'éveillait lucide comme si elle n'avait pas fermé l'œil.

Cela s'explique par le fait que le haut degré, qui fait passer d'une mesure quantitative relative à une mesure absolue, peut aussi être conçu comme une manière (de posséder la qualité considérée).

Par voie de conséquence, cette équivalence se retrouve dans le cas des comparaisons à parangon, même si (comme il a été dit plus haut) celles-ci sont exprimées prototypiquement par comme : plus blanc que neige/aussi blanc que neige/blanc comme neige sont équivalents.

A l'inverse, nous avons vu plus haut qu'avec une qualité gradable, l'identité (qualitative) de manière peut équivaloir à une égalité (quantitative) de degré : 


\section{Il est grand comme son frère/Il est aussi grand que son frère.}

Les constructions comparatives offrent encore d'autres glissements de la qualité vers la quantité, ainsi le marqueur autrement (littéralement 'd'une autre manière') concourt-il à l'expression d'une inégalité quantitative dans des exemples comme :

C'est un paresseux : sa femme travaille autrement que lui (= travaille à un autre degré, càd. beaucoup plus; et non pas 'd'une autre manière's)

Cette froide et ferme résolution, autrement rare chez nos jeunes Français que la bravoure armée ... (= beaucoup plus rare)

Vous saurez la soigner autrement mieux que ma mère (= beaucoup mieux).

\subsection{Comparaisons qualitatives}

Le chevillage par identification de la manière, qui est le propre du comme introduisant une subordonnée comparative à portée intraprédicative, est de nature à induire un certain nombre d'effets pragmatiques particuliers. Outre le haut degré et les comparaisons à parangon, sur lesquels nous ne reviendrons pas, il convient de signaler la diversité des effets de comme lorsque, en surface, il encadre deux noms ' $\mathrm{N}_{1}$ comme $\mathrm{N}_{2}$ '.

Nous laisserons de côté les cas où l'analyse conduit à traiter 'comme $\mathrm{N}_{2}$ ' en tant que subordonnée elliptique à portée extraprédicative. Il s'agit notamment de l'effet de coordination (où comme peut être reformulé par et), que l'on trouve dans des exemples comme :

Et l'un comme l'autre entra dans la voie des confidences

C'est là qu'il faut tout craindre : l'indifférence comme la partialité, la froideur comme la passion, le savoir comme l'ignorance.

Dans ce type d'exemples, la comparaison consiste en une analogie de situation, même si la subordonnée n'est pas explicitement détachée comme elle l'est dans :

\section{La Finlande, comme la Belgique, comporte deux éléments ethniques.}

Les effets que nous considèrerons sont ceux qui résultent d'une identité de 'manière d'être' (C. Fuchs \& P. Le Goffic $2005 \S 2$ ), c'est-à-dire qui chevillent la proposition matrice contenant $\mathrm{N}_{1}$ et la subordonnée contenant $\mathrm{N}_{2}$ autour d'un prédicat 'être' ('comme $\mathrm{N}_{2}$ ' ayant une fonction d'épithète par rapport à $\mathrm{N}_{1}$ ). La diversité des effets pragmatiques qui en résultent sont induits par les types de contextes.

\subsubsection{L'effet de qualification}

Exemple :

Une brise comme un souffle passait sur l'étang

càd. 'une brise (qui était) comme/de la manière dont (est) un souffle'; la subordonnée elliptique qualifie le type de 'brise'.

Ce fonctionnement est très proche de celui que l'on rencontre dans des structures du type :

\footnotetext{
${ }^{9}$ On retrouverait l'interprétation qualitative dans un exemple comme : Elle travaille le bois autrement que lui.
} 
C'est plein de livres comme tu les aimes

càd. 'de livres qui sont comme/de la manière dont tu aimes les livres' (avec un attribut de l'objet dans la subordonnée: 'tu aimes les livres d'une certaine manière', à l'instar de $t u$ aimes la soupe chaude). Sur ce type de structures, qui induit un effet caractérisant sur le $\mathrm{N}_{1}$, voir C. Fuchs \& P. Le Goffic (sous presse).

\subsubsection{L'effet d'identification}

Exemple :

Vous imaginez-vous, Monsieur de Pourceaugnac, qu'une fille comme la mienne soit si affamée de mari?

càd. 'une fille ${ }_{1}$ qui est comme ma fille $e_{2}$ est'. Ici, l'identité de manière d'être joue entre deux instances du même terme 'fille' qui, dans le contexte, sont comprises comme référant à une seule et même personne : la comparaison joue au niveau du «type ».

En revanche, la même séquence une fille comme la mienne pourrait, dans un autre contexte, être utilisée pour construire un effet de 'qualification'. Ainsi dans :

\section{Tu aurais bien voulu avoir une fille comme la mienne}

où les deux instances de 'fille' sont comprises comme renvoyant à deux entités distinctes : la comparaison joue au niveau du «t token ».

\subsubsection{L'effet d'exemplification}

Dans l'exemple :

\section{Certains conifères comme le mélèze perdent leurs feuilles en hiver}

càd. 'certains conifères qui sont comme est le mélèze', un effet d'exemplification est créé : 'le mélèze est un exemple/une instance de conifère'.

\subsubsection{L'effet d'approximation}

On arrive ici à un tyep d'effet «en bout de chaîne », le plus éloigné d'une comparaison prototypique. Exemples : famille)

Ils ont tous comme un air de famille (= quelque chose qui est comme est un air de

Il y a comme un défaut (= quelque chose qui est comme est un défaut)

Il a comme hésité (= il a fait quelque chose qui est comme est hésiter).

\section{Conclusion}

A l'issue de ce parcours, trois points nous semblent acquis en ce qui concerne les représentations sémantiques que l'on peut associer aux structures syntaxiques de comparaison en français.

Premièrement, le mécanisme de la comparaison opère toujours à propos d'entités : on compare deux entités distinctes, ou bien une seule entité considérée sous deux points de vue 
différents. Selon les cas, ces entités peuvent être co-planaires ou dissymétriques. L'identification des entités est parfois problématique.

Deuxièmement, le paramètre, qui constitue ce par rapport à quoi les entités sont comparées quantitativement ou qualitativement, est toujours de nature prédicative. La détermination de ce paramètre et de son empan ne peut se faire, lorsque la proposition matrice comporte plusieurs éléments de nature prédicative, que par une mise en parallèle de la structure de la proposition matrice et de celle de la subordonnée.

Troisièmement, la comparaison quantitative ('déclencheur $+q u e$ ') et la comparaison qualitative en comme ont des fonctionnements très différents et ne connaissent de recouvrements (zones d'équivalence) que locaux, sous certaines conditions très particulières.

\section{QEuvres citées}

Fournier, Nathalie; Catherine Fuchs. 1999. L'évolution du statut de faire dans les comparatives en comme et la constitution du groupe verbal $\left(17^{\grave{\mathrm{e}}}-20^{\mathrm{e}}\right.$ siècles). Verbum XXI : 3, Presses universitaires de Nancy, pp. 289-322.

Fournier, Nathalie; Catherine Fuchs. Sous presse(a). Que et comme comparatifs. Lexique 18, Lille : Presses de 1'Université de Lille.

Fournier, Nathalie; Catherine Fuchs. Sous presse(b). Les subordonnées comparatives détachées. In Les linguistiques du détachement, D. Apothéloz, B. Combettes, F. Neveu (eds.), Berne : Peter Lang.

Fuchs, Catherine; Pierre Le Goffic. 2005. La polysémie de comme. In La Polysémie, O. Soutet ed.), Paris : Presses de l'Université de Paris-Sorbonne, pp. 267-292.

Fuchs, Catherine; Pierre Le Goffic. Sous presse. Un emploi caractérisant de comme : Un de ces exemples comme on en trouve partout. Langue Française 157 (à paraître en mars 2008).

Haspelmath, Martin; Oda Buchholz. 1998. Equative and similative constructions in the languages of Europe. In Adverbial Constructions in the Languages of Europe, J. van der Auwera (ed.), Berlin/New-York : Mouton/de Gruyter, pp. 277-334.

Heine, Bernd. 1997. Cognitive Foundations of Grammar. Oxford/New-York: Oxford University Press.

Rivara, René. 1990. Le système de la comparaison. Paris : Minuit.

Rivara, René. 1995. Pourquoi il n'y a que deux relations de comparaison. Faits de langue 5, pp. 19-39.

Stassen, Leon. 1985. Comparison and Universal Grammar. Oxford/New-York: Basil Blackwell.

Ulland, Harald. 1993. Les nominalisations agentive et instrumentale en français moderne. Berne : Peter Lang.

Van Peteghem, Marleen. 2000. Les indéfinis corrélatifs: autre, même et tel. In De l'indétermination à la qualification: les indéfinis, L. Bosveld-de Smet, M. Van Peteghem, D. Van de Velde (eds), Presses de l'Université d'Artois, pp. 117-202.

\section{Summary}

This article deals with syntactic and semantic representation of comparative structures in French. We propose an analysis of quantitative comparatives (plus, moins, aussi ... que) and qualitative comparatives (comme) which highlights their common properties as well as their specificities. The first section $(\S 1)$ offers a syntactic typology of matrix clause structures and (comparative) subordinate clause structures. The following sections consider the various 
aspects of semantic representations, as related to syntactic structures : we successively deal with $(\S 2$.) the type of parameter, ( $\S 3$.) the type of differential constituant in the subordinate clause, (§ 4.) the type of parallel constituant in the matrix clause (with restitution of ellipses and anaphora), ( $\$ 5$.$) the type of compared terms, by contrasting quantitative comparisons and$ qualitative comparison, and ( $\S 6$.) the type of comparison, accounting for prototypical structures as well as for pragmatic effects induced by certain configurations.

Keywords : matrice, subordonnée, termes en $q u$-, anaphore, ellipse, paramètre, déclencheur, différentiel, constituant parallèle, comparé, prédicat, variable, quantitatif, qualitatif, échelle de degrés, pragmatique /matrix clause, subordinate clause, ${ }^{*} K^{w^{-}}$terms, anaphora, ellipsis, parameter, parameter marker, differential constituant, parallel constituant, comparee, predicate, variable, quantitative, qualitative, scale of degree, pragmatics.

Adresses des auteurs

Catherine Fuchs

LATTICE

ENS

1 rue Maurice Arnoux

92120 Montrouge

France

catherine.fuchs@ens.fr

Nathalie Fournier

Université Lumière-Lyon2

LESLA

18 Quai Claude Bernard

69007 Lyon

France

np.fournier@wanadoo.fr

Pierre Le Goffic

Université Paris 3 (Centre de Linguistique Française)

13 rue Santeuil,

F-75005 Paris

France

pierre.le-goffic@univ-paris3.fr 